\title{
DECOMPOSITIONS OF BANACH LATTICES INTO DIRECT SUMS
}

\author{
P. G. CASAZZA, N. J. KALTON AND L. TZAFRIRI
}

\begin{abstract}
We consider the problem of decomposing a Banach lattice $Z$ as a direct sum $Z=X \oplus Y$ where $X$ and $Y$ are complemented subspaces satisfying a condition of incomparability (e.g. every operator from $Y$ to $X$ is strictly singular). We treat both the atomic and nonatomic cases. In particular we answer a question of Wojtaszczyk by showing that $L_{1} \oplus L_{2}$ has unique structure as a nonatomic Banach lattice.
\end{abstract}

One of the most important problems in the theory of Banach lattices, which is still open, is whether any complemented subspace of a Banach lattice must be linearly isomorphic to a Banach lattice. The main difficulty seems to lie in the fact that most of the criteria for a Banach space to be isomorphic to a lattice do not really distinguish between lattices and their complemented subspaces.

We do not actually treat this question in the present paper but rather consider the situation $Z=X \oplus Y$, where $Z$ is a Banach lattice and $X$ and $Y$ two complemented subspaces which are assumed to satisfy different conditions that make them "distinct" in some or another sense. This line of research was initiated by P. Wojtaszczyk [28] (and also by I. S. Edelstein and P. Wojtaszczyk [3]) who proved that if $Z$ has a normalized unconditional basis $\left\{z_{n}\right\}_{n=1}^{\infty}$ (i.e. it is a separable atomic lattice) so that every linear operator from $Y$ into $X$ is compact then $\left\{z_{n}\right\}_{n=1}^{\infty}$ splits into two disjoint parts which are respectively equivalent to bases of $X$ and $Y$. In particular, both $X$ and $Y$ have unconditional bases. The proof of this result is based on a fundamental theorem from [28 and 3], which is mentioned below as Theorem A. We give here a different proof which does not make use of Theorem A but instead is based on a simple "change of signs" result from [2], which is described below as Theorem B. We also consider the case when the compactness assumption above is replaced by the total incomparability of $X$ and $Y$ for which we prove a similar result provided $X$ and $Y$ have unconditional bases. Unfortunately, the most interesting case when every operator from $Y$ into $X$ is assumed to be strictly singular (which was raised as an open problem in [28]) remains unsolved. We conclude the section devoted to the atomic case with a simple theorem on block bases of a space with unconditional basis $\left\{z_{n}\right\}_{n=1}^{\infty}$ whose span is complemented. Such a block basis splits into two disjoint parts, the first equivalent to a subsequence of $\left\{z_{n}\right\}_{n=1}^{\infty}$

Received by the editors September 5, 1986.

1980 Mathematics Subject Classification (1985 Revision). Primary 46B30, 46B15; Secondary $46 \mathrm{~A} 40$.

This research was supported by Grant No. 84-00210 from the United States-Israel Binational Science Foundation (BSF), Jerusalem, Israel.

The research of the first author was also supported in part by NSF Grant DMS 8500938 .

The research of the second author was also supported in part by NSF Grant DMS 8601401. 
and the second equivalent to a sequence in the complement of the span of the block basis.

In the continuous case we show that a nonatomic Banach lattice $Z$ which has some nontrivial cotype cannot be split into totally incomparable infinite dimensional subspaces; thus if $Z \approx X \oplus Y$ with $X, Y$ totally incomparable then either $\operatorname{dim} X<$ $\infty$ or $\operatorname{dim} Y<\infty$. The same result, under the assumption that every operator $T: X \rightarrow Y$ is strictly singular, is false as the example $L_{1} \oplus L_{2}$ shows. However, in this case we give some partial results which suggest that the general result may be true if $Z$ has some nontrivial type.

We conclude by studying the structure of Banach lattices of the form $L_{1} \oplus X$. where $X$ is reflexive. We show that if $Z$ is a Banach lattice isomorphic to $L_{1} \oplus Y$, where $Y$ has some nontrivial type then $Z$ has a band decomposition $Z \approx Z_{0} \oplus Z_{0}^{\perp}$, where $Z_{0}$ is an AL-space and $Z_{0}^{\perp}$ is isomorphic to $Y$. As a consequence the Banach space $L_{1} \oplus L_{2}$ has a unique structure as a nonatomic Banach lattice; this answers a question raised by $\mathrm{P}$. Wojtaszczyk [28].

0. Preliminaries. In order to make the paper as self-contained as possible, we quote in this section some results that are used very often. We begin with a result which is crucial all throughout the article; instead of presenting the two available versions separately we incorporate them as one theorem.

THEOREM A $[\mathbf{3}, \mathbf{2 8}]$. Let $X$ and $Y$ be two Banach spaces and suppose that they are either totally incomparable (i.e. no infinite dimensional subspace of $X$ is isomorphic to a subspace of $Y$ ) or that every bounded linear operator $T$ from $Y$ into $X$ is strictly singular (i.e. there exists no infinite dimensional subspace of $Y$ so that $T$ restricted to $Y$ is an isomorphism). Let $V$ be a complemented subspace of $X \oplus Y$. Then there exists an automorphism $\psi$ of $X \oplus Y$ such that $\psi(V)=X_{1} \oplus Y_{1}$, where $X_{1}=\psi(V) \cap X$ and $Y_{1}=\psi(V) \cap Y$ are complemented subspaces of $X$, respectively $Y$.

The first section of the paper is devoted to spaces with an unconditional basis. The standard material on such spaces can be found e.g. in [15]. We quote here a decomposition theorem which is used several times.

THEOREM B [2]. Let $X$ and $Y$ be two Banach spaces and $Q$ a bounded linear projection from $X \oplus Y$ onto a subspace $V$ with a finite or infinite $K$-unconditional basis $\left\{v_{n}\right\}_{n \in \tau}$ so that

$$
Q(u)=\sum_{n \in \tau} v_{n}^{*}(u) v_{n}
$$

where $\left\{v_{n}^{*}\right\}_{n \in \tau}$ are functionals biorthogonal to $\left\{v_{n}\right\}_{n \in \tau}$. Let $P_{X}$ and $P_{Y}$ denote the corresponding projections from $X \oplus Y$ onto $X$, respectively $Y$. Fix $0<\alpha<1$ and split $\tau$ into two disjoint subsets $\tau_{X}$ and $\tau_{Y}$ so that $v_{n}^{*}\left(Q P_{X} v_{n}\right) \geq \alpha$, for $n \in \tau_{X}$ and $v_{n}^{*}\left(Q P_{Y} v_{n}\right) \geq 1-\alpha$, for $n \in \tau_{Y}$.

Then there exists a constant $M=M(\|Q\|, K, \alpha)$ so that, for any choice of $1 \leq$ $p \leq \infty,\left\{v_{n}\right\}_{n \in \tau_{X}}$ and $\left\{v_{n}\right\}_{n \in \tau_{Y}}$ are respectively $M$-equivalent to $\left\{r_{n}(t) P_{X} v_{n}\right\}_{n \in \tau_{X}}$ and $\left\{r_{n}(t) P_{Y} v_{n}\right\}_{n \in \tau_{Y}}$, considered as elements of $L_{p}(X)$ and $L_{p}(Y)$. Moreover, $\left[r_{n}(t) P_{X} v_{n}\right]_{n \in \tau_{X}}$ and $\left[r_{n}(t) P_{Y} v_{n}\right]_{n \in \tau_{Y}}$ are $M$-complemented in $L_{p}(X)$, respectively $L_{p}(Y)$. 
Here, as usual, $\left\{r_{n}(t)\right\}_{n=1}^{\infty}$ denotes the sequence of the Rademacher functions and $L_{p}(X)$ stands for $L_{p}([0,1], X)$.

The spaces with an unconditional basis can be viewed as atomic lattices. In the second and third sections, we study nonatomic and also general lattices. The standard material on this topic is available, for instance, in [16 and 26]. Of particular interest are the order continuous Banach lattices, i.e. those in which any decreasing net $\left\{z_{\gamma}\right\}$ whose g.l.b. is 0 satisfies $\lim _{\gamma} z_{\gamma}=0$. Order continuous Banach lattices with a weak unit admit a very useful representation theorem (cf. e.g. [16, 1.b.14]).

THEOREM C. Let $Z$ be an order continuous Banach lattice with a weak unit. Then there exist a probability space $(\Omega, \Sigma, \mu)$, an (not necessarily closed) ideal $\tilde{Z}$ of $L_{1}(\Omega, \Sigma, \mu)$ and a lattice norm $\|\cdot\|_{\tilde{Z}}$ on $\tilde{Z}$ such that

(i) $Z$ is order isometric to $\tilde{Z}$.

(ii) $\tilde{Z}$ is dense in $L_{1}(\Omega, \Sigma, \mu)$ and $L_{\infty}(\Omega, \Sigma, \mu)$ is dense in $\tilde{Z}$.

(iii) $\|f\|_{1} \leq\|f\|_{\tilde{Z}} \leq 2\|f\|_{\infty} ; f \in L_{\infty}(\Omega, \Sigma, \mu)$.

(iv) The dual of the above order isometry maps $Z^{*}$ onto the Banach lattice $\tilde{Z}^{*}$ of all $g$ for which

$$
\|g\|_{\tilde{Z}^{*}}=\sup \left\{\int_{\Omega} f g d \mu ;\|f\|_{\tilde{Z}} \leq 1\right\}<\infty
$$

and $g(f)=\int_{\Omega} f g d \mu$.

A lattice of functions as above will be called a Banach function space.

We point out that a Banach lattice which contains no subspace isomorphic to $c_{0}$ is order continuous. In fact, such a lattice has the stronger property that any increasing net $\left\{z_{\gamma}\right\}_{\gamma \in \Gamma}$ with $\sup _{\gamma \in \Gamma}\left\|z_{\gamma}\right\|<\infty$ converges to its l.u.b.

As we have already mentioned, the third section studies embedding of $L_{1}$-spaces into Banach lattices. One of the most important tools used in this section is a representation theorem for operators from $L_{1}(0,1)$ to a Banach lattice containing no copy of $c_{0}$, which is presented and used extensively in [10 and 11]. Rather than discussing these results in detail, we collect here only those facts that are needed in the sequel. We also quote a theorem concerning the so-called sign-preserving operators.

An operator $T$ from $L_{1}(0,1)$ into a Banach space $Z$ is called sign-preserving provided there exist a $\delta>0$ and a subset $A$ of $[0,1]$ of positive measure so that $\|T(\psi)\| \geq \delta$, whenever $\psi \in L_{1}(0,1)$ has mean zero and $|\psi|=\chi_{A}\left(\chi_{A}\right.$ denotes the characteristic function of $A$ ).

THEOREM D. Let $Z$ be a Banach lattice containing no isomorphic copy of $c_{0}$.

(i) [11] If $T$ is an isomorphism from $L_{1}(0,1)$ into $Z$ then there exists also an order isomorphism $S$ from $L_{1}(0,1)$ into $Z$.

(ii) $[11,25]$ An operator $S$ from $L_{1}(0,1)$ into a $L_{1}(\Omega, \Sigma, \mu)$-space is a lattice homomorphism if and only if there exist measurable functions $0 \leq a: \Omega \rightarrow \mathbf{R}$ and $\sigma: \Omega \rightarrow[0,1]$ so that

$$
S(\psi)(\omega)=a(\omega) \psi(\sigma(\omega), \quad \omega \in \Omega \text { a.e. }
$$

for all $\psi \in L_{1}(0,1)$. 
(iii) [7] If $T$ is a sign-preserving operator from $L_{1}(0,1)$ into $Z$ then $T$ is an isomorphism on some subspace of $L_{1}(0,1)$ which is isomorphic to $L_{1}(0,1)$.

Additional details on operators from $L_{1}$-spaces into Banach lattices can be found in $[10,11,24,25,7,4$ and 5$]$.

1. Spaces with an unconditional basis (atomic lattices). We start this section by presenting an alternative proof of P. Wojtaszczyk's decomposition theorem from [28]. The original proof relies on Theorem A while ours uses instead Theorem B whose proof is considerably simpler than that of Theorem A.

THEOREM 1.1. Let $X$ and $Y$ be two Banach spaces such that every bounded linear operator from $Y$ into $X$ is compact. Then every unconditional basis $\left\{z_{n}\right\}_{n=1}^{\infty}$ of $X \oplus Y$ splits into two disjoint parts $\left\{z_{n}\right\}_{n \in C_{1}}$ and $\left\{z_{n}\right\}_{n \in C_{2}}$ that are respectively equivalent to bases of $X$ and $Y$. In particular, $X$ and $Y$ have unconditional bases.

PROOF. We can assume without loss of generality that $\left\|z_{n}\right\|=1$, for all $n$, and that the direct sum $X \oplus Y$ is taken in the sense of $l_{1}$. In this case, the corrresponding projections $P_{X}$ and $P_{Y}$ from $X \oplus Y$ onto $X$, respectively $Y$, are of norm one. For each subset $C$ of the integers, let $Q_{C}$ denote the projection defined by

$$
Q_{C}\left(\sum_{n=1}^{\infty} a_{n} z_{n}\right)=\sum_{n \in C} a_{n} z_{n}
$$

for any choice of $\left\{a_{n}\right\}_{n=1}^{\infty}$. Put $K=\sup \left\|Q_{C}\right\|$.

Split now the integers into two disjoint subsets $A_{1}$ and $A_{2}$ such that $n \in A_{1} \Rightarrow$ $x_{n}^{*} x_{n} \geq \frac{1}{2}$ and $n \in A_{2} \Rightarrow y_{n}^{*} y_{n} \geq \frac{1}{2}$, where $x_{n}=P_{X} z_{n}, y_{n}=P_{Y} z_{n}, x_{n}^{*}=$ $P_{n}^{*} z_{n}^{*}, y_{n}^{*}=P_{Y}^{*} z_{n}^{*}$ and $\left\{z_{n}^{*}\right\}_{n=1}$ are the biorthogonal functionals associated to $\left\{z_{n}\right\}_{n=1}^{\infty}$.

By Theorem B, we conclude the existence of a constant $M=M(K)$ so that

$$
M^{-1}\left\|\sum_{i \in A_{1}} a_{i} z_{i}\right\| \leq \int_{0}^{1}\left\|\sum_{i \in A_{1}} a_{i} r_{i}(t) x_{i}\right\| d t \leq M\left\|\sum_{i \in A_{1}} a_{i} z_{i}\right\|
$$

and

$$
M^{-1}\left\|\sum_{i \in A_{2}} a_{i} z_{i}\right\| \leq \int_{0}^{1}\left\|\sum_{i \in A_{2}} a_{i} r_{i}(t) y_{i}\right\| d t \leq M\left\|\sum_{i \in A_{2}} a_{i} y_{i}\right\|,
$$

for any choice of scalars $\left\{a_{i}\right\}_{i=1}^{\infty}$. Similar inequalities hold in the dual.

LEMMA 1.2. There exists a subset $B_{1}$ of $A_{1}$ so that $\left\{z_{n}\right\}_{n \in B_{1}}$ is equivalent to $\left\{x_{n}\right\}_{n \in B_{1}}$ and $\left|A_{1} \sim B_{1}\right|$ is finite.

PROOF. Suppose the assertion of Lemma 1.2 is false. Since

$$
\left\|\sum_{i \in A_{1}} a_{i} x_{i}\right\| \leq\left\|\sum_{i \in A_{1}} a_{i} z_{i}\right\|
$$

for any choice of $\left\{a_{i}\right\}$, we can easily construct a sequence $\left\{C_{j}\right\}_{j=1}^{\infty}$ of mutually disjoint finite subsets of $A_{1}$ and vectors $u_{j}=\sum_{i \in C_{j}} b_{i} z_{i}$ such that $\left\|u_{j}\right\|=1$ and 
$\left\|P_{X} u_{j}\right\|<2^{-(j+1)}$, for all $j$. The inequalities mentioned above show the existence of signs $\varepsilon_{i}= \pm 1, i \in C_{j}, j=1,2, \ldots$, such that

$$
\left\|\sum_{i \in C_{j}} \varepsilon_{i} b_{i} x_{i}\right\| \geq M^{-1} .
$$

Consider now the automorphism $T_{\varepsilon}$ of $X \oplus Y$ defined by $T_{\varepsilon} z_{i}=\varepsilon_{i} z_{i}$, for all $i$, where $\varepsilon_{i}$ is chosen as above if $i \in C_{j}$, for some $j$, or $\varepsilon_{i}=+1$, otherwise. Since the operator $P_{X} T_{\varepsilon \mid Y}: Y \rightarrow X$ must be compact it follows by passing to a subsequence if necessary that $P_{X} T_{\varepsilon} P_{Y} u_{j} \rightarrow x$, as $j \rightarrow \infty$, for some $x \in X$. Since $\| u_{j}-$ $P_{Y} u_{j} \|<2^{-(j+1)}$ we also get that $P_{X} T_{\varepsilon} u_{j} \rightarrow x$, as $j \rightarrow \infty$. On the other hand, $P_{X} T_{\varepsilon} u_{j}=\sum_{i \in C_{j}} \varepsilon_{i} b_{i} x_{i}$ and therefore $\|x\| \geq M^{-1}$. This shows that the sequence $\left\{u_{j}\right\}_{j=1}^{\infty}$ does not tend weakly to zero. Hence, there is a functional $u^{*}$ on $X \oplus Y$ and a subsequence $\left\{u_{j(k)}\right\}_{k=1}^{\infty}$ of $\left\{u_{j}\right\}_{j=1}$ so that $\inf _{k} u^{*} u_{j(k)}>0$. It follows that $\left\{u_{j(k)}\right\}_{k=1}^{\infty}$ is equivalent to the unit vector basis of $l_{1}$, and, moreover, that $\left[u_{j(k)}\right]_{k=1}^{\infty}$ is complemented in $X \oplus Y$. A standard perturbation argument shows that there is a $l_{0}$ so that $\left[P_{Y} u_{j(k)}\right]_{k \geq l_{0}}$ is complemented in $Y$ and isomorphic to $l_{1}$. This means that there are quotient maps from $Y$ onto $X$ which, of course, is a contradiction.

LEMMA 1.3. There exists a subset $B_{2}$ of $A_{2}$ so that $\left\{z_{n}\right\}_{n \in B_{2}}$ is equivalent to $\left\{y_{n}\right\}_{n \in B_{2}}$ and $\left|A_{2} \sim B_{2}\right|$ is finite.

PROOF. If the assertion of Lemma 1.3 is false then one can construct exactly as in the proof of Lemma 1.2 a sequence of mutually disjoint finite subsets $\left\{D_{j}\right\}_{j=1}^{\infty}$ of $A_{2}$ and vectors $v_{j}=\sum_{i \in D_{j}} d_{i} z_{i}$ such that $\left\|v_{j}\right\|=1$ and $\left\|P_{Y} v_{j}\right\|<2^{-(j+1)}$, for all $j$.

If $\left\{v_{j}\right\}_{j=1}^{\infty}$ does not tend weakly to zero then it contains a subsequence which is equivalent to the unit vector basis of $l_{1}$. By Theorem B, the subspace $\left[r_{n}(t) y_{n}\right]_{n \in A_{2}}$ of $L_{2}(Y)$ also contains a subspace isomorphic to $l_{1}$. Hence, by G. Pisier [20], we conclude that $Y$ contains an isomorphic copy of $l_{1}$. A standard gliding hump argument shows that one can find in $Y$ a sequence of elements which, on one hand, is equivalent to the unit vector basis of $l_{1}$ and, on the other hand, is equivalent to a block basis of $\left\{z_{n}\right\}_{n=1}^{\infty}$. Therefore, $Y$ contains a complemented copy of $l_{1}$ and we can easily construct noncompact operators from $Y$ into $X$. This contradiction shows that $v_{j} \stackrel{w}{\rightarrow} 0$, as $j \rightarrow \infty$.

Now for each $j$, let $v_{j}^{*} \in X^{*}$ be so that $\left\|v_{j}^{*}\right\|=1$ and $v_{j}^{*}\left(P_{X} v_{j}\right) \geq 1-2^{-(j+1)}$. It follows that $v_{j}^{*}\left(v_{j}\right) \geq 1-2^{-j}$, for all $j$. By passing to a subsequence if needed, we can assume without loss of generality that $v_{j}^{*} \stackrel{w^{*}}{\rightarrow} v^{*}$, as $j \rightarrow \infty$, for some $v^{*} \in X^{*}$. Since $v^{*}\left(v_{j}\right) \rightarrow 0$, as $j \rightarrow \infty$, we can choose an integer $j_{1}$ so that $\left|v^{*}\left(v_{j}\right)_{1}\right|<1 / 8$. Then we can find an integer $i_{1}$ so that $\left|v_{i_{1}}^{*}\left(v_{j_{1}}\right)\right|<1 / 8$. Put $w_{1}^{*}=v_{j_{1}}^{*}-v_{i_{1}}^{*}$ and notice that $w_{1}^{*}\left(v_{j_{1}}\right) \geq 1-1 / 2^{j}-1 / 8>1 / 4$. Repeating this procedure, we construct a subsequence $\left\{v_{j_{k}}\right\}_{k=1}^{\infty}$ of $\left\{v_{j}\right\}_{j=1}^{\infty}$ and a sequence $\left\{w_{k}^{*}\right\}_{k=1}^{\infty}$, which are differences of the $v_{j}^{*}$ 's and therefore $w^{*}-\lim _{k \rightarrow \infty} w_{k}^{*}=0$, such that $w_{k}^{*}\left(v_{j_{k}}\right)>1 / 4$, for all $k$. By a gliding hump argument, we can assume without loss of generality that

$$
w_{k}^{*}=\sum_{i \in \tilde{D}_{k}} c_{i} z_{i}^{*}+\tilde{w}_{k}^{*},
$$


where $\left\{\tilde{D}_{k}\right\}_{k=1}^{\infty}$ are mutually disjoint finite subsets of the integers, $\left\{c_{i}\right\}$ suitable scalars and $\left\{\tilde{w}_{k}^{*}\right\}_{k=1}^{\infty}$ a sequence which tends in norm to zero. Therefore, we will assume that $\left\|\tilde{w}_{k}^{*}\right\|<1 / 8$, for all $k$. It follows that

$$
\frac{1}{4}<w_{k}^{*}\left(v_{j_{k}}\right) \leq\left(\sum_{i \in \tilde{D}_{k}} c_{i} z_{i}^{*}\right)\left(\sum_{i \in D_{j_{k}}} d_{i} z_{i}\right)+\frac{1}{8}
$$

which implies that

$$
\left\|\sum_{i \in \tilde{D}_{k} \cap D_{j_{k}}} c_{i} z_{i}^{*}\right\| \geq \frac{1}{8}
$$

for all $k$. By Theorem B, applied to the dual of $X \oplus Y$, we find signs $\varepsilon_{i}= \pm 1, i \in$ $\tilde{D}_{k} \cap D_{j_{k}}, k=1,2, \ldots$ so that

$$
\left\|\sum_{i \in \tilde{D}_{k} \cap D_{j_{k}}} \varepsilon_{i} c_{i} y_{i}^{*}\right\|>\frac{1}{8 M}
$$

for all $k$.

Let now $\tilde{T}_{\varepsilon}$ be the linear operator on $X \oplus Y$ which is defined by $\tilde{T}_{\varepsilon} z_{i}=\varepsilon_{i} z_{i}$, if $i \in \tilde{D}_{k} \cap D_{j_{k}}$ for some $k$, or by $\tilde{T}_{\varepsilon} z_{i}=0$, otherwise. Since $P_{X} \tilde{T}_{\varepsilon} P_{Y}$ is compact it follows that its adjoint $P_{Y}^{*} \tilde{T}_{\varepsilon}^{*} P_{X}^{*}$ is also compact and thus, we can assume without loss of generality that $P_{Y}^{*} \tilde{T}_{\varepsilon}^{*} P_{X}^{*} w_{k}^{*} \rightarrow 0$, as $k \rightarrow \infty$. Since $w_{k}^{*} \in X^{*}$, for all $k$, we get that

$$
\sum_{i \in \tilde{D}_{k} \cap D_{j_{k}}} \varepsilon_{i} c_{i} y_{i}^{*}=P_{Y}^{*} \tilde{T}_{\varepsilon}^{*}\left(\sum_{i \in \tilde{D}_{k}} c_{i} z_{i}^{*}\right) \rightarrow 0,
$$

as $k \rightarrow \infty$, and this, of course, is a contradiction.

LEMMA 1.4. There exists a subset $D$ of the integers such that

(i) $Y=\left[y_{n}\right]_{n \in D}$.

(ii) $\left\{z_{n}\right\}_{n \in D}$ is equivalent to $\left\{y_{n}\right\}_{n \in D}$.

PROOF. Suppose that the complement $B_{2}^{c}$ of $B_{2}$ in $\mathbb{N}$ consists of the integers $\left\{n_{1}<n_{2}<\cdots<n_{j}<\cdots\right\}$. Put $E_{0}=B_{2}$. If $y_{n_{1}} \in\left[y_{n}\right]_{n \in E_{0}}$ then omit this vector; if $y_{n_{1}} \notin\left[y_{n}\right]_{n \in E_{0}}$ then set $E_{1}=B_{2} \cup\left\{n_{1}\right\}$. A simple verification shows that $\left\{z_{n}\right\}_{n \in E_{1}}$ is equivalent to $\left\{y_{n}\right\}_{n \in E_{1}}$. Indeed, suppose that there exists a vector $\sum_{n \in E_{1}} a_{n} z_{n} \neq 0$ so that $\sum_{n \in E_{1}} a_{n} y_{n}=0$. By our assumption on $y_{n_{1}}$, we conclude that the coefficient of $y_{n_{1}}$ must be zero. Hence, $\sum_{n \in E_{0}} a_{n} y_{n}=0$ which, by Lemma 3 , implies that $\sum_{n \in E_{0}} a_{n} z_{n}=0$, a contradiction.

We continue this procedure with $n_{2}, n_{3}, \ldots$ and construct subsets $E_{2}, E_{3}, \ldots$ of the integers as above. If the argument stops after $m$ steps then we put $D=E_{m}$ and the proof is completed since $\left[y_{n}\right]_{n \in E_{m}}$ must be equal to $Y$. On the other hand, if this procedure can be repeated as many times as we like, then there exists a sequence $\left\{E_{j}\right\}_{j=1}^{\infty}$ of subsets of the integers so that

(a) $B_{2}=E_{0} \subset E_{1} \subset \cdots \subset E_{j} \subset \cdots$.

(b) $\left[y_{n}\right]_{n \in E_{j-1}} \neq\left[y_{n}\right]_{n \in E_{j}} \neq Y$, for all $j$.

(c) $\left[y_{n}\right]_{n \in \cup_{j=1}^{\infty}} E_{j}=Y$. 
Hence, there exist vectors $y_{j}^{\prime} \in Y$ such that $\left\|y_{j}^{\prime}\right\|=1$ and $d\left(y_{j}^{\prime},\left[y_{n}\right]_{n \in E_{j-1}}\right) \geq \frac{1}{2}$, for all $j \geq 1$. Since, by our assumption, the restriction of the operator $P_{X} Q_{B_{1}}$ to $Y$ is compact it follows, by passing to a subsequence if necessary, that $P_{X} Q_{B_{1}} y_{j}^{\prime} \rightarrow x$, as $j \rightarrow \infty$, for some $x \in X$. Suppose now that

$$
y_{j}^{\prime}=\sum_{n=1}^{\infty} a_{n}^{j} z_{n}=\sum_{n=1}^{\infty} a_{n}^{j} y_{n}, \quad j=1,2, \ldots
$$

Then

$$
\lim _{j \rightarrow \infty} \sum_{n \in B_{1}} a_{n}^{j} x_{n}=x .
$$

But, by Lemma $2,\left\{z_{n}\right\}_{n \in B_{1}}$ is equivalent to $\left\{x_{n}\right\}_{n \in B_{1}}$. Hence,

$$
\lim _{j \rightarrow \infty} \sum_{n \in B_{1}} a_{n}^{j} y_{n}=y
$$

for some $y \in Y$. Since

$$
B_{1}^{c} \sim B_{2}=\left(A_{1} \sim B_{1}\right) \cup\left(A_{2} \sim B_{2}\right)
$$

there exists an integer $l$ so that $\left[y_{n}\right]_{n \in B_{1}^{c}} \subset\left[y_{n}\right]_{n \in E_{k}}$, whenever $k \geq l$. It follows that, for $j>k \geq l$, we have

$$
d\left(\sum_{n \in B_{1}} a_{n}^{j} y_{n},\left[y_{n}\right]_{n \in E_{k}}\right)=d\left(y_{j}^{\prime},\left[y_{n}\right]_{n \in E_{k}}\right) \geq d\left(y_{j}^{\prime},\left[y_{n}\right]_{n \in E_{j-1}}\right) \geq \frac{1}{2}
$$

This implies that $d\left(y,\left[y_{n}\right]_{n \in E_{k}}\right) \geq 1 / 2$ for all $k$. Hence, $d(y, Y) \geq 1 / 2$ which, of course, is contradictory.

We return now to the Proof of Theorem 1.1. Let $D$ be the set constructed in Lemma 1.4 and notice that if $x \in X \cap\left[z_{n}\right]_{n \in D}$ then $x=\sum_{n \in D} d_{n} z_{n}=\sum_{n \in D} d_{n} x_{n}$ for a suitable sequence of scalars $\left\{d_{n}\right\}_{n \in D}$. Hence, $\sum_{n \in D} a_{n} y_{n}=0$ and, by Lemma 1.4 , also $x=0$. Thus, $X \cap\left[z_{n}\right]_{n \in D}=\{0\}$. Actually, $X+\left[z_{n}\right]_{n \in D}$ is a closed subspace of $X \oplus Y$. Indeed, if there exist sequences $\left\{u_{k}\right\}_{k=1}^{\infty}$ in $X$ and $\left\{v_{k}\right\}_{k=1}^{\infty}$ in $\left[z_{n}\right]_{n \in D}$ with $\left\|u_{k}\right\|=\left\|v_{k}\right\|=1$, for all $k$, and $\lim _{k \rightarrow \infty}\left\|u_{k}+v_{k}\right\|=0$ then $\lim _{k \rightarrow \infty}\left\|u_{k}+P_{X} v_{k}\right\|=0$. Let $S$ be the isomorphism frorn $Y$ onto $\left[z_{n}\right]_{n \in D}$ given by Lemma 1.4. Then $P_{X} S$ is a compact operator which maps $S^{-1} v_{k}$ into $P_{X} v_{k}$, for all $k$. Hence, $\left\{P_{X} v_{k}\right\}_{k=1}^{\infty}$ and thus also $\left\{u_{k}\right\}_{k=1}^{\infty}$ contain convergent subsequences and this contradicts the fact that $X \cap\left[z_{n}\right]_{n \in D}=\{0\}$.

Observe now that $Y$ is contained in the internal direct sum $X \oplus\left[z_{n}\right]_{n \in D}$ since $Y=\left[y_{n}\right]_{n \in D}$ and, for $m \in D, y_{m}=z_{m}-x_{m} \in X \oplus\left[z_{n}\right]_{n \in D}$. Therefore,

$$
\left[z_{n}\right]_{n=1}^{\infty}=X \oplus Y=X \oplus\left[z_{n}\right]_{n \in D}
$$

which readily implies that $X$ is isomorphic to $\left[z_{n}\right]_{n \in D^{c}}$. This completes the proof since we already know that $Y$ is isomorphic to $\left[z_{n}\right]_{n \in D}$, by Lemma 1.4 .

P. Wojtaszczyk raised in [28] the question whether Theorem 1 remains valid when the assumption that all the operators from $Y$ into $X$ are compact is replaced by the weaker condition that all the operators from $Y$ into $X$ are strictly singular. This question seems to be still open. As far as we know, it is still unknown if the above compactness condition can be replaced by the total incomparability of $X$ and $Y$. We prove such a result under some additional assumptions. 
THEOREM 1.5. Let $X$ and $Y$ be two totally incomparable Banach spaces having each an unconditional basis. Then every unconditional basis $\left\{z_{n}\right\}_{n=1}^{\infty}$ of $X \oplus Y$ splits into two disjoint parts which are respectively equivalent to bases of $X$ and $Y$.

PROOF. We shall maintain the notation used in the proof of Theorem 1.1 as well as the basic assumptions made there. We need first a lemma.

LEMMA 1.6. If there exists an $\alpha>0$ so that $x_{n}^{*}\left(x_{n}\right) \geq \alpha$, for all $n$, then

(i) $\left\{z_{n}\right\}_{n=m}^{\infty}$ is equivalent to $\left\{x_{n}\right\}_{n=m}^{\infty}$, for some integer $m$.

(ii) $Y$ is finite dimensional.

ProOF. By Theorem $\mathrm{B}$, there is a $M=M(K, \alpha)$ so that

$$
M^{-1}\left\|\sum_{n=1}^{\infty} c_{n} z_{n}\right\| \leq \int_{0}^{1}\left\|\sum_{n=1}^{\infty} c_{n} r_{n}(t) x_{n}\right\| d t \leq M\left\|\sum_{n=1}^{\infty} c_{n} z_{n}\right\|
$$

for any choice of $\left\{c_{n}\right\}_{n=1}^{\infty}$. A similar inequality holds in the dual situation for $\left\{x_{n}^{*}\right\}_{n=1}^{\infty}$ and $\left\{z_{n}^{*}\right\}_{i=1}^{\infty}$.

Let now $\left\{\xi_{i}\right\}_{i=1}^{\infty}$ be a normalized unconditional basis for $X$ and let $\left\{\xi_{i}^{*}\right\}_{i=1}^{\infty}$ be the corresponding biorthogonal functionals. For $x=\sum_{i=1}^{\infty} b_{i} \xi_{i}$ put

$$
|x|=\sum_{i=1}^{\infty}\left|b_{i}\right| \xi_{i} \quad \text { and } \quad\left|x_{i}\right|^{2}=\sum_{i=1}^{\infty}\left|b_{i}\right|^{2} \xi_{i}
$$

Then, for any choice of $\left\{c_{n}\right\}_{n=1}^{\infty}$, we have

$$
M\left\|\sum_{n=1}^{\infty} c_{n} z_{n}\right\| \geq\left\|\int_{0}^{1}\left|\sum_{n=1}^{\infty} c_{n} r_{n}(t) x_{n}\right| d t\right\| \geq \frac{1}{\sqrt{2}}\left\|\left(\sum_{n=1}^{\infty}\left|c_{n} x_{n}\right|^{2}\right)^{1 / 2}\right\| .
$$

Since

$$
P f=\sum_{n=1}^{\infty}\left(\int_{0}^{1} x_{n}^{*}(f(s)) r_{n}(s) d s\right) \frac{r_{n}(t) x_{n}}{x_{n}^{*}\left(x_{n}\right)}, \quad f \in L_{2}(X),
$$

defines a projection of norm $\leq M$ from $L_{2}(X)$ onto $\left[r_{n}(t) x_{n}\right]_{n=1}^{\infty}$ and since, as above,

$$
M \sqrt{2}\left\|\sum_{n=1}^{\infty} d_{n} z_{n}^{*}\right\| \geq\left\|\left(\sum_{n=1}^{\infty}\left|d_{n} x_{n}^{*}\right|^{2}\right)^{1 / 2}\right\|
$$

for any choice of $\left\{d_{n}\right\}_{n=1}^{\infty}$, we can apply a standard duality argument and conclude that

$$
\left\|\sum_{n=1}^{\infty} c_{n} z_{n}\right\| \leq M^{4} \sqrt{2}\left\|\left(\sum_{n=1}^{\infty}\left|c_{n} x_{n}\right|^{2}\right)^{1 / 2}\right\|,
$$

for every choice of scalars $\left\{c_{n}\right\}_{n=1}^{\infty}$.

Suppose now that either (i) or (ii) do not hold. In this case, it is easily verified that there are integers $p_{0}=1<p_{1}<p_{2}<\cdots<p_{j}<\cdots$ and blocks $u_{j}=$ $\sum_{i=p_{j-1}+1}^{p_{j}} a_{i} z_{i}$ so that $\left\|u_{j}\right\|=1$ and $\left\|P_{X} u_{j}\right\|<2^{-(j+1)}$, for all $j$.

Notice that $u_{j} \stackrel{w}{\rightarrow} 0$, as $j \rightarrow \infty$. Indeed, if this is false then we can assume without loss of generality that $\left\{u_{j}\right\}_{j=1}^{\infty}$ is equivalent to the unit vector basis of $l_{1}$. Moreover, $\left[u_{j}\right]_{j=1}^{\infty}$ is complemented in $X \oplus Y$. In view of the condition satisfied by 
$\left\{P_{X} u_{j}\right\}_{j=1}^{\infty}$, it follows that $Y$ contains a complemented copy of $l_{1}$. Since $\left\{z_{n}\right\}_{n=1}^{\infty}$ is equivalent to $\left\{r_{n}(t) x_{n}\right\}_{n=1}^{\infty}$, considered e.g. as a sequence in $L_{2}(X)$, we conclude that also $L_{2}(X)$ contains a complemented subspace isomorphic to $l_{1}$. By [20], $X$ contains a copy of $l_{1}$, too, and this contradicts the assumption that $X$ and $Y$ are totally incomparable.

Now, for each $j$, put

$$
s_{j}=\left(\sum_{n=p_{j-1}+1}^{p_{j}}\left|a_{n} x_{n}\right|^{2}\right)^{1 / 2} \in X
$$

Then, for every $i$, we have

$$
\lim _{j \rightarrow \infty} \xi_{i}^{*}\left(s_{j}\right)=0 .
$$

Indeed, if for some fixed $i$ there exists a $\beta>0$ so that $\xi_{i}^{*}\left(s_{j}\right) \geq \beta$, for all $j$, then

$$
\left(\int_{0}^{1}\left|\sum_{n=p_{j-1}+1}^{p_{j}} a_{n} r_{n}(t) \xi_{i}^{*}\left(x_{n}\right)\right|^{2} d t\right)^{1 / 2}=\left(\sum_{n=p_{j-1}+1}^{p_{j}}\left|a_{n} \xi_{i}^{*}\left(x_{n}\right)\right|^{2}\right)^{1 / 2} \geq \beta .
$$

Hence, there are signs $\varepsilon_{n}= \pm 1, p_{j-1}<n \leq p_{j}$, such that

$$
\left(P_{X}^{*} \xi_{i}^{*}\right)\left(\sum_{n=p_{j-1}+1}^{p_{j}} a_{n} \varepsilon_{n} z_{n}\right)=\xi_{i}^{*}\left(\sum_{n=p_{j-1}+1}^{p_{j}} a_{n} \varepsilon_{n} x_{n}\right) \geq \beta
$$

for all $j$. This contradicts the fact that $\mathrm{w}-\lim _{j \rightarrow \infty} u_{j}=0$. The condition satisfied by $\left\{s_{j}\right\}_{j=1}^{\infty}$ shows that there is no loss of generality in assuming that $\left\{s_{j}\right\}_{j=1}^{\infty}$ is actually a block basis of $\left\{\xi_{i}\right\}_{i=1}^{\infty}$ (note that, by the inequalities established above, $1 / M^{4} \sqrt{2} \leq\left\|s_{j}\right\| \leq M \sqrt{2}$, for all $j$ ). These inequalities also yield that, for any choice of $\left\{b_{j}\right\}_{j=1}^{\infty}$, we have

$$
M \sqrt{2}\left\|\sum_{j=1}^{\infty} b_{j} u_{j}\right\| \geq\left\|\left(\sum_{j=1}^{\infty}\left|b_{j}\right|^{2} \sum_{n=p_{j-1}+1}^{p_{j}}\left|a_{n} x_{n}\right|^{2}\right)^{1 / 2}\right\|=\left\|\sum_{j=1}^{\infty}\left|b_{j}\right| s_{j}\right\|
$$

and, also

$$
\left\|\sum_{j=1}^{\infty} b_{j} u_{j}\right\| \leq M^{4} \sqrt{2}\left\|\sum_{j=1}^{\infty}\left|b_{j}\right| s_{j}\right\| .
$$

It follows that $\left[u_{j}\right]_{j=1}^{\infty}$ is isomorphic to the subspace $\left[s_{j}\right]_{j=1}^{\infty}$ of $X$. However, the fact that $\left\|P_{X} u_{j}\right\|<2^{-(j+1)}$, for all $j$, implies that $\left[u_{j}\right]_{j=1}^{\infty}$ is isomorphic to a subspace of $Y$. This, of course, contradicts the total incomparability of $X$ and $Y$.

We return to the Proof of Theorem 1.5. Let $A_{1}$ and $A_{2}$ be defined as in the proof of Theorem 1.1. By Theorem A, there exists an automorphism $\psi: X \oplus Y \rightarrow X \oplus Y$ such that

$$
\psi\left(\left[z_{n}\right]_{n \in A_{1}}\right)=X_{1} \oplus Y_{1},
$$

where $X_{1}=\psi\left(\left[z_{n}\right]_{n \in A_{1}}\right) \cap X$ and $Y_{1}=\psi\left(\left[z_{n}\right]_{n \in A_{1}}\right) \cap Y$ are complemented subspaces of $X$, respectively $Y$. 
Put $\hat{z}_{n}=\psi\left(z_{n}\right) ; n=1,2, \ldots$, and let $\left\{\hat{z}_{n}^{*}\right\}_{n=1}^{\infty}$ be the functionals biorthogonal to $\left\{\hat{z}_{n}\right\}_{n=1}^{\infty}$. In general, the set

$$
\hat{A}_{1}=\left\{n \in \mathbb{N} ; \hat{z}_{n}^{*}\left(P_{X} z_{n}\right) \geq \frac{1}{2}\right\}
$$

need not coincide with $A_{1}$. However, $A_{1}$ and $\hat{A}_{1}$ can differ only by a finite number of elements. Indeed, if e.g. $\hat{A}_{1} \cap A_{2}$ contains an infinite sequence $\left\{n_{i}\right\}_{i=1}^{\infty}$ and $\left\{z_{n_{i}}\right\}_{i=1}^{\infty}$ does not tend weakly to zero then, by Theorem B, both $L_{2}(X)$ and $L_{2}(Y)$ must contain an isomorphic copy of $l_{1}$. By [20], so do $X$ and $Y$ and this is a contradiction. If, on the other hand, $z_{n_{i}} \stackrel{w}{\rightarrow} 0$ then we can assume without loss of generality that $\left\{y_{n_{i}}\right\}_{i=1}^{\infty}$ and $\left\{P_{X} \hat{z}_{n_{i}}\right\}_{i=1}^{\infty}$ are mutually disjoint blocks on $\left\{z_{n}\right\}_{n=1}^{\infty}$, respectively $\left\{\hat{z}_{n}\right\}_{n=1}^{\infty}$. Hence, again by Theorem B, $\left\{y_{n_{i}}\right\}_{i=1}^{\infty}$ is equivalent to $\left\{z_{n_{i}}\right\}_{i=1}^{\infty}$ and $\left\{P_{X} \hat{z}_{n_{i}}\right\}_{i=1}^{\infty}$ to $\left\{\hat{z}_{n_{i}}\right\}_{i=1}^{\infty}$; i.e. $X$ and $Y$ are not totally incomparable.

It follows from these considerations that $\psi\left(\left[z_{n}\right]_{n \in A_{1} \cap \hat{A}_{1}}\right)$ is a space of finite codimension in $X_{1} \oplus Y_{1}$, and

$$
\psi\left(\left[z_{n}\right]_{n \in A_{1} \cap \hat{A}_{1}}\right)=\hat{X}_{1} \oplus \hat{Y}_{1},
$$

where $\hat{X}_{1}$ and $\hat{Y}_{1}$ are spaces of finite codimension in $X_{1}$, respectively $Y_{1}$. By Lemma 1.6, $Y_{1}$ must be finite dimensional and thus $k=\operatorname{dim} Y_{1}<\infty$.

Suppose now that $X=X_{1} \oplus X_{2}$ and $Y=Y_{1} \oplus Y_{2}$. Then $\psi\left(\left[z_{n}\right]_{n \in A_{2}}\right)$ is clearly isomorphic to $X_{2} \oplus Y_{2}$. In view of the total symmetry of our assumptions, we can conclude, as above, that $m=\operatorname{dim} X_{2}<\infty$. If e.g. $m \geq k$ then we switch $m-k$ vectors from $A_{2}$ to $A_{1}$ thus obtaining new sets $C_{1}$ and $C_{2}$. It is easily checked that $\psi\left(\left[z_{n}\right]_{n \in C_{1}}\right)$ is isomorphic to $X$ and $\psi\left(\left[z_{n}\right]_{n \in C_{2}}\right)$ to $Y$.

REMARK. It is not clear if the assumption made in the statement of Theorem 1.5 that $X$ and $Y$ have unconditional bases is actually needed. As is well known, the problem whether every complemented subspace of a space with an unconditional basis must have itself an unconditional basis is still unsolved. Theorem 1.5 could perhaps provide some means to construct a counterexample since it specifies in a precise manner an unconditional basis for $X$ whenever $X \oplus Y$ has an unconditional basis and $X$ and $Y$ are totally incomparable.

In order to prove results on splitting of bases of a direct sum, one has to make some additional assumptions, otherwise, the result clearly fails. This fact is put in evidence by a simple example pointed out by P. Wojtaszczyk in [28]. He noticed that, for $1<p \neq 2, L_{p}(0,1)$ is isomorphic to $L_{p}(0,1) \oplus l_{2}$ but the image under any isomorphism of the normalized Haar basis in $L_{p}(0,1)$ is an unconditional basis of $L_{p}(0,1) \oplus l_{2}$ which does not split as e.g. in Theorem 1.1 .

In the simpler case when one of the factors of the direct sum is $c_{0}$ or $l_{p}$, the situation is considerably clearer, as shown by the next result.

THEOREM 1.7. Let $X$ and $Y$ be two Banach spaces and suppose that $X$ is isomorphic to $c_{0}$ or $l_{p}$, for some $p \geq 1$. If $X \oplus Y$ has an unconditional basis then so does $Y$.

PROOF. The cases when $X$ is isomorphic either to $c_{0}$ or to $l_{1}$ can be treated separately. For instance, suppose that $X$ is isomorphic to $c_{0}$ and that $X \oplus Y$ has a normalized unconditional basis $\left\{z_{n}\right\}_{n=1}^{\infty}$. If every operator from $X$ into $Y$ is compact then we conclude that $Y$ has an unconditional basis, by using Theorem 1 . On the other hand, if there exists a noncompact operator $T$ from $c_{0}$ into $Y$ then 
we can find a $\gamma>0$, a normalized block basis $\left\{u_{n}\right\}_{n=1}^{\infty}$ of the unit vector basis of $c_{0}$ and a block basis $\left\{v_{n}\right\}_{n=1}^{\infty}$ of $\left\{z_{n}\right\}_{n=1}^{\infty}$ so that $\left\|v_{n}\right\| \geq \gamma$ and $\left\|T u_{n}-v_{n}\right\| \leq 2^{-n}$, for all $n$. It follows easily that $\left\{v_{n}\right\}_{n=1}^{\infty}$ is equivalent to the unit vector basis of $c_{0}$ and therefore $Y$ contains a complemented copy of $c_{0}$. Hence, $X \oplus Y$ is isomorphic to $Y$ and this completes the proof of this case.

We pass now to the case when $X$ is isomorphic to $l_{p}$, for some $p>1$. We shall assume that every bounded linear operator from $Y$ into $X$ is strictly singular; otherwise, $Y$ contains a complemented subspace isomorphic to $l_{p}$ and thus $Y$ is isomorphic to $Y \oplus X$ which has an unconditional basis. We shall keep the notations and the conventions introduced throughout the proof of Theorem 1.1.

We consider first the vectors $\left\{z_{n}\right\}_{n \in A_{1}}$. By Theorem $\mathrm{B},\left[z_{n}\right]_{n \in A_{1}}$ is isomorphic to $\left[r_{n}(t) x_{n}\right]_{n \in A_{1}}$, considered e.g. as a complemented subspace of $L_{p}(X)$ which, in turn, is isomorphic to $L_{p}(0,1)$. By Theorem $\mathrm{A},\left[z_{n}\right]_{n \in A_{1}}$ is isomorphic to a direct sum of the form $X_{1} \oplus Y_{1}$, where $X_{1}$ and $Y_{1}$ are complemented subspaces of $X$, respectively $Y$. It follows that $Y_{1}$ is either isomorphic to $l_{2}$ or is an $\mathcal{L}_{p}$-space. In the case when $Y_{1}$ is an infinite dimensional $\mathcal{L}_{p}$-space it contains a complemented copy of $l_{p}$ (cf. [14]) and thus, there exist nonstrictly singular operators from $Y$ into $X$. Therefore, either $Y_{1}$ is isomorphic to $l_{2}$ or it is finite dimensional. In both these cases, $Y_{1}$ has an unconditional basis.

We proceed now with $\left[z_{n}\right]_{n \in A_{2}}$. Let $X_{2}$ and $Y_{2}$ be such that $X=X_{1} \oplus X_{2}$ and $Y=Y_{1} \oplus Y_{2}$. Then, by Theorem A, $\left[z_{n}\right]_{n \in A_{2}}$ is isomorphic to $X_{2} \oplus Y_{2}$. If $\operatorname{dim} X_{2}<\infty$ then $Y_{2}$ is of finite codimension in a space with an unconditional basis and therefore it has itself, an unconditional basis. Suppose now that $X_{2}$ is isomorphic to $l_{p}$. In this case, we can find a block basis $u_{j}=\sum_{n=q_{j-1}+1}^{q_{j}} a_{n} z_{n}, j=$ $1,2, \ldots$, of $\left\{z_{n}\right\}_{n \in A_{2}}$ so that

(a) $\left\|u_{j}\right\|=1$, for all $j$.

(b) $\left\{u_{j}\right\}_{j=1}^{\infty}$ is equivalent to the unit vector basis of $l_{p}$.

(c) $\left\{u_{j}\right\}_{j=1}^{\infty}$ is equivalent to a sequence in $X$.

(d) $\left[u_{j}\right]_{j=1}^{\infty}$ is a complemented subspace in $\left[z_{n}\right]_{n \in A_{2}}$.

Let $R$ be a projection from $\left[z_{n}\right]_{n \in A_{2}}$ onto $\left[u_{j}\right]_{j=1}^{\infty}$. We can assume without loss of generality that

$$
R(z)=\sum_{j=1}^{\infty} u_{j}^{*}(z) u_{j}, \quad z \in\left[z_{n}\right]_{n \in A_{2}}
$$

where $u_{j}^{*}=\sum_{n=q_{j-1}+1}^{q_{j}} b_{n} z_{n}^{*}$, for all $j$, and $\left\{b_{n}\right\}_{n \in A_{2}}^{\infty}$ are suitable scalars so that $b_{n} a_{n} \geq 0$, for all $n$. Notice that $\left\{u_{j}^{*}\right\}_{j=1}^{\infty}$ is equivalent to the unit vector basis of $l_{q}$, where $q=p /(p-1)$. Since

$$
\begin{gathered}
\int_{0}^{1}\left(\sum_{n=q_{j-1}+1}^{q_{j}} b_{n} r_{n}(t) y_{n}^{*}\right)\left(\sum_{n=q_{j-1}+1}^{q_{j}} a_{n} r_{n}(t) y_{n}\right) d t \\
=\sum_{n=q_{j-1}+1}^{q_{j}} a_{n} b_{n} y_{n}^{*}\left(y_{n}\right) \geq \frac{1}{2} u_{j}^{*}\left(u_{j}\right)
\end{gathered}
$$


for all $j$, we conclude the existence of signs $\varepsilon_{n}= \pm 1, q_{j-1}<n \leq q_{j}, j=1,2, \ldots$, so that

$$
\left(\sum_{n=q_{j-1}+1}^{q_{j}} b_{n} \varepsilon_{n} y_{n}^{*}\right)\left(\sum_{n=q_{j-1}+1}^{q_{j}} a_{n} \varepsilon_{n} y_{n}\right) \geq \frac{1}{2}
$$

for all $j$. We write now

$$
v_{j}=\sum_{n=q_{j-1}+1}^{q_{j}} a_{n} \varepsilon_{n} y_{n} \quad \text { and } \quad v_{j}^{*}=\sum_{n=q_{j-1}+1}^{q_{j}} b_{n} \varepsilon_{n} y_{n}^{*}, \quad j=1,2, \ldots
$$

By switching to a subsequence, we may assume with no loss of generality that $\left\{v_{j}\right\}_{j=1}^{\infty}$ and $\left\{v_{j}^{*}\right\}_{j=1}^{\infty}$ are block bases of $\left\{z_{n}\right\}_{n=1}^{\infty}$, respectively $\left\{z_{n}^{*}\right\}_{n=1}^{\infty}$. Hence,

$\left(\mathrm{a}^{1}\right) v_{j}^{*}\left(v_{j}\right) \geq \frac{1}{2}$ and $v_{j}^{*}\left(v_{h}\right)=0$, for all $j$ and all $h \neq j$. $l_{q}$.

$\left(\mathrm{b}^{1}\right)\left\{v_{j}\right\}_{j=1}^{\infty}$ is dominated by the unit vector basis of $l_{p}$ and $\left\{v_{j}^{*}\right\}_{j=1}^{\infty}$ by that of

It follows easily, by a simple duality argument, that $\left\{v_{j}\right\}_{j=1}^{\infty}$ is equivalent to the unit vector basis of $l_{p}$ and $\left\{v_{j}^{*}\right\}_{j=1}^{\infty}$ to that of $l_{q}$. Moreover,

$$
R^{1}(y)=\sum_{j=1}^{\infty} \frac{v_{j}^{*}(y)}{v_{j}^{*}\left(v_{j}\right)} v_{j}, \quad y \in Y
$$

defines a bounded projection from $Y$ onto $\left[v_{j}\right]_{j=1}^{\infty}$. This means that $Y$ contains a complemented copy of $l_{p}$ and thus there are nonstrictly singular operators from $Y$ into $X$.

Since both $Y_{1}$ and $Y_{2}$ have unconditional bases it follows that so does $Y$.

We conclude this section with a result on block bases of an unconditional basis which span a complemented subspace.

THEOREM 1.8. Let $Z$ be a Banach space with a normalized unconditional basis $\left\{z_{n}\right\}_{n=1}^{\infty}$ and let $\left\{x_{j}\right\}_{j=1}^{\infty}$ be a normalized block basis of $\left\{z_{n}\right\}_{n=1}^{\infty}$. Suppose that $Z=\left[x_{j}\right]_{j=1}^{\infty} \oplus Y$, for some subspace $Y$ of $Z$. Then there is a partition of the integers into two subsets $A$ and $B$ so that

(i) $\left\{x_{j}\right\}_{j \in A}$ is equivalent to a subsequence of $\left\{z_{n}\right\}_{n=1}^{\infty}$.

(ii) $\left[x_{j}\right]_{j \in B}$ is isomorphic to a complemented subspace of $Y$.

PrOOF. Denote by $K$ the unconditionality constant of $\left\{z_{n}\right\}_{n=1}^{\infty}$ and suppose that

$$
x_{j}=\sum_{n=p_{j-1}+1}^{p_{j}} a_{n} z_{n}
$$

for a suitable sequence of scalars $\left\{a_{n}\right\}_{n=p_{j-1}+1}^{p_{j}}, j=1,2, \ldots$, and for integers $p_{0}=1<p_{1}<p_{2}<\cdots$. Let $P$ be a bounded projection from $Z$ onto $\left[x_{j}\right]_{j=1}^{\infty}$. As in the proof of Theorem 1.7, we an assume without loss of generality that

$$
P(z)=\sum_{j=1}^{\infty} x_{j}^{*}(z) x_{j}, \quad z \in Z,
$$

where $x_{j}^{*}=\sum_{n=p_{j-1}+1}^{p_{j}} b_{n} z_{n}^{*}, j=1,2, \ldots$, are functionals biorthogonal to $\left\{x_{j}\right\}_{j=1}^{\infty}$ and $\left\{b_{n}\right\}_{n=p_{j-1}+1}^{p_{j}}, j=1,2, \ldots$, are suitable scalars so that $a_{n} b_{n} \geq 0$, for all 
$n\left(\left\{z_{n}^{*}\right\}_{n=1}^{\infty}\right.$ denotes, as usual, the sequence of the biorthogonal functions associated with $\left.\left\{z_{n}\right\}_{n=1}^{\infty}\right)$.

Put

$$
A=\left\{j \in \mathbb{N} ; \sup _{p_{j-1}<n \leq p_{j}} a_{n} b_{n}>1 / 2\right\}
$$

and $B=\mathbb{N} \sim A$. We prove first that $\left\{x_{j}\right\}_{j \in A}$ is equivalent to $\left\{z_{n(j)}\right\}_{j \in A}$, where, for each $j \in A, n(j)$ is selected to be one of the indices satisfying $p_{j-1}<n(j) \leq p(j)$ and $a_{n(j)} b_{n(j)}>1 / 2$. Notice that $\left|a_{n(j)}\right| \leq K$ and $\left|b_{n(j)}\right| \leq\|P\|$, for all $j$. Hence, for any choice of scalars $\left\{c_{j}\right\}_{j \in A}$, we have

$$
\begin{aligned}
\|P\| \cdot\left\|\sum_{j \in A} c_{j} z_{n(j)}\right\| & \geq\left\|\sum_{j \in A} c_{j} P\left(z_{n(j)}\right)\right\|=\left\|\sum_{j \in A} c_{j} b_{n(j)} x_{j}\right\| \geq \frac{1}{4 K^{2}}\left\|\sum_{j \in A} c_{j} x_{j}\right\| \\
& \geq \frac{1}{4 K^{3}}\left\|\sum_{j \in A} c_{j} a_{n(j)} z_{n(j)}\right\| \geq \frac{1}{16 K^{5}}\left\|\sum_{j \in A} c_{j} z_{n(j)}\right\|,
\end{aligned}
$$

and this completes the proof of (i).

We consider now the set $B$. For $j \in B$ and $p_{j-1}<n \leq p_{j}$, we have $0 \leq a_{n} b_{n} \leq$ $1 / 2$. Thus, we can choose signs $\varepsilon_{n}= \pm 1$ so that

$$
\omega_{j}=\sum_{n=p_{j-1}+1}^{p_{j}} \varepsilon_{n} a_{n} b_{n}
$$

satisfies $\left|\omega_{j}\right| \leq 1 / 2$, for all $j$. Put

$$
y_{j}=\sum_{n=p_{j-1}+1}^{p_{j}} \varepsilon_{n} a_{n}(I-P) z_{n} \in Y
$$

and observe that

$$
y_{j}=\sum_{n=p_{j-1}+1}^{p_{j}} \varepsilon_{n} a_{n} z_{n}-\omega_{j} x_{j}=\sum_{n=p_{j-1}+1}^{p_{j}}\left(\varepsilon_{n}-\omega_{j}\right) a_{n} z_{n},
$$

for all $j$. Hence, for every choice of scalars $\left\{d_{j}\right\}_{j \in B}$, we get

$$
\begin{aligned}
K\left\|\sum_{j \in B} d_{j} x_{j}\right\| & \geq\left\|\sum_{j \in B} d_{j} \sum_{n=p_{j-1}+1}^{p_{j}} \varepsilon_{n} a_{n} z_{n}\right\| \geq \frac{1}{\|I-P\|}\left\|\sum_{j \in B} d_{j} y_{j}\right\| \\
& =\frac{1}{\|I-P\|}\left\|\sum_{j \in B} d_{j} \sum_{n=p_{j-1}+1}^{p_{j}}\left(\varepsilon_{n}-\omega_{j}\right) a_{n} z_{n}\right\| \\
& \geq \frac{1}{4 K\|I-P\|}\left\|\sum_{j \in B} d_{j} x_{j}\right\|
\end{aligned}
$$

since $\left|\varepsilon_{n}-\omega_{j}\right| \geq 1 / 2$, for all $p_{j-1}<n \leq p_{j}, j=1,2, \ldots$. 
Finally, in order to prove that $\left[y_{j}\right]_{j=1}^{\infty}$ is complemented in $Y$, we notice that the operator $Q$, defined by,

$$
Q(z)=\sum_{j=1}^{\infty}\left(\sum_{n=p_{j-1}+1}^{p_{j}} l_{n} b_{n} c_{n}\right) y_{j}, \quad z=\sum_{i=1}^{\infty} c_{i} z_{i} \in Z,
$$

where $l_{n}=1 /\left(\varepsilon_{n}-\omega_{j}\right), p_{j-1}<n \leq p_{j}, j=1,2, \ldots$, is a bounded linear projection from $Z$ onto $\left[y_{j}\right]_{j=1}^{\infty}$.

REMARK. The assumption made in Theorem 1.8 that $\left[x_{j}\right]_{j=1}^{\infty}$ is a block basis of $\left\{z_{n}\right\}_{n=1}^{\infty}$ is not redundant. Indeed, take as $Z$ the direct sum $l_{p} \oplus l_{1}, 1<p \neq 2$, and as $\left\{z_{n}\right\}_{n=1}^{\infty}$ the union of the unit vector bases of $l_{p}$ and $l_{1}$. Let $\left\{v_{j}\right\}_{j=1}^{\infty}$ denote the unit vector basis of the space $\left(\sum_{n=1}^{\infty} \bigoplus l_{2}^{n}\right) p$ and $T_{p}$ an isomorphism from this space onto $l_{p}$. Then clearly the sequence $x_{j}=T_{p}\left(v_{j}\right), j=1,2, \ldots$, cannot be split as in the statement of Theorem 1.8.

2. Nonatomic lattices. In this section we shall consider some continuous versions of the results of section one. We show that if $Z$ is a nonatomic Banach lattice with some nontrivial cotype and $Z=X \oplus Y$ where $X$ and $Y$ are totally incomparable then either $\operatorname{dim} X<\infty$ or $\operatorname{dim} Y<\infty$. Some similar but rather less complete results are obtained for the situation when every operator $T: X \rightarrow Y$ is strictly singular. We give an example to show that the cotype assumption is necessary and prove a lattice analogue of Theorem 1.7.

Let us state first our main result on totally incomparable subspaces.

THEOREM 2.1. Let $Z$ be a nonatomic Banach lattice with nontrivial cotype and suppose $Z=X \oplus Y$ where $X$ and $Y$ are totally incomparable subspaces of $Z$. Then either $\operatorname{dim} X<\infty$ or $\operatorname{dim} Y<\infty$.

Theorem 2.1 is an immediate consequence of Theorem 2.2(a).

THEOREM 2.2. Let $Z$ be a nonatomic Banach lattice with nontrivial cotype, and suppose $Z=X \oplus Y$ where every bounded operator $T: X \rightarrow Y$ is strictly singular. Then each of the following conditions implies $\operatorname{dim} X<\infty$ :

(a) $X$ contains no subspace isomorphic to $l_{2}$.

(b) $Z$ has nontrivial type and $X$ contains no complemented subspace isomorphic to $l_{2}$.

(c) $Z$ has nontrivial type and $Y$ contains a complemented infinite dimensional subspace with an unconditional basis.

The proof of Theorem 2.2 involves a series of lemmas. The consequence of Lemma 2.3 is that we need only consider the case when $Z$ is a Banach function space as described in $\S 0$.

LEMMA 2.3. Let $Z$ be an order-continuous Banach lattice and suppose $Z=$ $X \oplus Y$ where $X$ and $Y$ are infinite dimensional subspaces of $Z$ such that every bounded operator $T: X \rightarrow Y$ is strictly singular. Then there is a band $Z_{0}$ in $Z$ with a weak order-unit such that $Z_{0}=X_{0} \oplus Y_{0}$, where $X_{0}$ and $Y_{0}$ are, respectively, infinite-dimensional complemented subspaces of $X$ and $Y$.

If, in addition, $Y$ contains a complemented subspace with an unconditional basis, then we can further assume that $Y_{0}$ has a complemented subspace with unconditional basis. 
ProOF. Let $X^{\prime}$ and $Y^{\prime}$ be separable infinite dimensional subspaces of $X$ and $Y$, respectively. Then there is a band $Z_{0}$ with a weak order-unit containing $X^{\prime}$ and $Y^{\prime}$. As $Z_{0}$ is complemented by a band projection, $P_{0}$ say, we can use Theorem A to deduce that $Z_{0}=X_{0} \oplus Y_{0}$, where $X_{0}$ and $Y_{0}$ are complemented subspaces of $X$ and $Y$, respectively. If $P_{Y}$ is the projection of $Z$ onto $Y$ then $P_{Y \mid Z_{0}}$ is not strictly singular so that $\operatorname{dim} Y_{0}=\infty$ (since $P_{Y \mid Z_{0}}$ is identity on $Y^{\prime}$ ). Similarly $P_{X \mid Z_{0}}$ is not strictly singular so that $\operatorname{dim} X_{0}=\infty$.

For the last part we may assume $Y^{\prime}$ is complemented and has an unconditional basis. Then $Y^{\prime}$ is complemented in $Z_{0}$ and so $Y^{\prime}=X_{1} \oplus Y_{1}$, where $X_{1}$ is complemented in $X_{0}$ and $Y_{1}$ is complemented in $Y_{0}$. Clearly we must have $\operatorname{dim} X_{1}<\infty$ and so $Y_{1}$ has an unconditional basis.

LEMMA 2.4. Let $Z$ be an order-continuous Banach function space on a nonatomic probability measure space $(\Omega, \Sigma, \mu)$. Suppose $f \in Z$ with $f \neq 0$.

(a) There is a sequence $\left\{f_{n}\right\} \in Z$ with $\left|f_{n}\right|=|f|$, for all $n$ and $f_{n} \stackrel{w}{\rightarrow} \mathbf{0}$, as $n \rightarrow \infty$.

(b) If $Z$ has nontrivial cotype then $\left\{f_{n}\right\}_{n=1}^{\infty}$ may be chosen in (a) to be a basic sequence equivalent to the standard $l_{2}$-basis.

(c) If $Z$ has nontrivial type then $\left\{f_{n}\right\}_{n=1}^{\infty}$ may be chosen in (b) so that $\left[f_{n}\right]_{n=1}^{\infty}$ is, in addition, complemented in $Z$.

ProOF. We first prove (b). If $Z$ has nontrivial cotype then the map

$$
A: L_{\infty}(\Omega, \Sigma, \mu) \rightarrow Z
$$

given by $A(g)=|f| g$ is $q$-absolutely summing for some $2<q<\infty$ (cf. [18]). By the Pietsch Factorization Theorem (cf. [15, 2.b.2]) there is a positive linear functional $F$ on $L_{\infty}$ with $F\left(\chi_{\Omega}\right)=1$ and a constant $C$ so that

$$
\||f| g\| \leq C\left(F\left(|g|^{q}\right)\right)^{1 / q} .
$$

Clearly we can assume $F$ is $\mu$-continuous so that for some $h \in L_{1}(\mu), h \geq 0$ and $\int_{\Omega} h d \mu=1$, we have

$$
\||f| g\| \leq C\left(\int_{\Omega}|g|^{q} h d \mu\right)^{1 / q} .
$$

Now, by Liapunoff's theorem $[16,2 . e .8]$, we can find $\Omega_{n, j} \in \Sigma$ for $n=0,1,2, \ldots$, $j=1,2, \ldots, 2^{n}$ with $\Omega_{0,1}=\Omega$ so that

(i) $\Omega_{n, 2 j-1} \cup \Omega_{n, 2 j}=\Omega_{n-1, j}$.

(ii) $\int_{\Omega_{n, j}}|f| d \mu=2^{-n} \int_{\Omega}|f| d \mu$.

(iii) $\int_{\Omega_{n, j}}^{n} h d \mu=2^{-n}$.

Let $\phi_{n}=\sum_{j=1}^{2^{n}}(-1)^{j} \chi_{\Omega_{n, j}}$ and let $f_{n}=|f| \phi_{n}$. Then, by Khintchine's inequality, $\left\{f_{n}\right\}_{n=1}^{\infty}$ is equivalent in $L_{1}(\mu)$ to the $l_{2}$-basis, and so

$$
\begin{aligned}
\left\|\sum_{n=1}^{\infty} a_{n} f_{n}\right\| & \geq \int_{\Omega}\left|\sum_{n=1}^{\infty} a_{n} f_{n}\right| d \mu=\int_{\Omega}\left|\sum_{n=1}^{\infty} a_{n} \phi_{n}\right||f| d \mu \\
& \geq \frac{1}{\sqrt{2}}\left(\sum_{n=1}^{\infty}\left|a_{n}\right|^{2}\right)^{1 / 2} .
\end{aligned}
$$


On the other hand,

$$
\begin{aligned}
\left\|\sum_{n=1}^{\infty} a_{n} f_{n}\right\| & \leq C\left(\int_{\Omega}\left|\sum_{n=1}^{\infty} a_{n} \phi_{n}\right|^{q} h d \mu\right)^{1 / q} \\
& \leq C^{\prime}\left(\sum_{n=1}^{\infty}\left|a_{n}\right|^{2}\right)^{1 / 2}
\end{aligned}
$$

by Khintchine's inequality in $L_{q}(h d \mu)$, when $C^{\prime}$ is a suitable constant. Thus (b) is established.

Note that (a) follows by constructing $\Omega_{n, j}$ to verify only (i) and (ii). Then $f_{n} \rightarrow 0$ weakly in $L_{1}(\mu)$ as $n \rightarrow \infty$ and since $\left\{f_{n}\right\}_{n=1}^{\infty}$ is relatively weakly compact in $Z$ we have also $f_{n} \rightarrow 0$ weakly in $Z$, as $n \rightarrow \infty$.

For (c) note that $Z$ is a $p$-convex lattice for some $p>1$, and the inclusion $Z \rightarrow L_{1}(\mu)$ is thus a $p$-convex operator. Thus, by $[\mathbf{1 2}]$ (cf. $\left.[\mathbf{1 6}, 1 . d .12]\right)$, the inclusion can be factored through an $L_{p}$-space. Then there exist operators $A_{1}: Z \rightarrow$ $L_{p}(\nu), A_{2}: L_{p}(\nu) \rightarrow L_{1}(\mu)$ so that $A_{2} A_{1}(f)=f$. In particular, $\left\{A_{1}\left(f_{n}\right)\right\}_{n=1}^{\infty}$ is a basic sequence equivalent to the $l_{2}$-basis in $L_{p}(\nu)$ and so has a subsequence $\left\{A_{1}\left(f_{n_{k}}\right)\right\}_{k=1}^{\infty}$, whose span is complemented. Then $\left[f_{n_{k}}\right]_{k=1}^{\infty}$ is also complemented in $Z$.

Before proceeding with the main part of the proof we introduce a concept which will be useful and make some preliminary observations. Let $\left\{u_{n}\right\}_{n=1}^{\infty}$ and $\left\{v_{n}\right\}_{n=1}^{\infty}$ be any two basic sequences in $Z$. We say $\left\{u_{n}\right\}_{n=1}^{\infty}$ and $\left\{v_{n}\right\}_{n=1}^{\infty}$ are strongly equivalent if there exist operators $S: Z \rightarrow Z$ and $T: Z \rightarrow Z$ with $S u_{n}=v_{n}, T v_{n}=u_{n}$. Note that strong equivalence implies equivalence. Note also that if $X$ and $Y$ are complemented subspaces of $Z$ and $\left\{u_{n}\right\}_{n=1}^{\infty} \subset X,\left\{v_{n}\right\}_{n=1}^{\infty} \subset Y$ are strongly equivalent then there exist nonstrictly singular operators from $X$ into $Y$ and from $Y$ into $X$.

We shall use an easy perturbation argument repeatedly. Let us suppose $\left\{u_{n}\right\}_{n=1}^{\infty}$ is a basic sequence so that $0<\inf _{n}\left\|u_{n}\right\| \leq \sup _{n}\left\|u_{n}\right\|<\infty$. Suppose $\left\{v_{n}\right\}_{n=1}^{\infty}$ is a sequence in $Z$ and $A: Z \rightarrow Z, B: Z \rightarrow Z$ are operators such that $\left\|u_{n}-B\left(v_{n}\right)\right\| \rightarrow 0$ and $\left\|v_{n}-A\left(u_{n}\right)\right\| \rightarrow 0$, as $n \rightarrow \infty$. Then there are subsequences $\left\{u_{n_{k}}\right\}_{k=1}^{\infty}$ and $\left\{v_{n_{k}}\right\}_{k=1}^{\infty}$ which are strongly equivalent basic sequences. In particular this holds when $A=B=I$.

LEMMA 2.5. Let $Z$ be a Banach function space on $(\Omega, \Sigma, \mu)$ with nontrivial type. Let $\left\{f_{n}\right\}_{n=1}^{\infty} \subset Z$ be a normalized unconditional basic sequence such that $\left[f_{n}\right]_{n=1}^{\infty}$ is complemented in $Z$. Then either $\left\{f_{n}\right\}_{n=1}^{\infty}$ is equivalent to the standard $l_{2}$-basis or $\left\{f_{n}\right\}_{n=1}^{\infty}$ has a subsequence $\left\{f_{n_{k}}\right\}_{k=1}^{\infty}$ which is strongly equivalent to a disjoint sequence $\left\{h_{k}\right\}_{k=1}^{\infty}$ in $Z$.

REMARK. Indeed $\left[h_{k}\right]_{k=1}^{\infty}$ is complemented.

PrOOF. Let $\left\{f_{n}^{*}\right\}_{n=1}^{\infty}$ be the dual basis in $Z^{*}$, so that the projection $P: Z \rightarrow$ $\left[f_{n}\right]_{n=1}^{\infty}$ is given by

$$
P(g)=\sum_{n=1}^{\infty}\left(\int_{\Omega} f_{n}^{*} \cdot g d \mu\right) f_{n} .
$$

Note that $Z$ is reflexive so that $Z^{*}$ is also order-continuous. We distinguish three cases. 
(1) Suppose

$$
\inf _{n} \int_{\Omega}\left|f_{n}\right| d \mu=0 .
$$

Then, by passing to a subsequence, we may suppose

$$
\lim _{n \rightarrow \infty} \int_{\Omega}\left|f_{n}\right| d \mu=0
$$

and, for each $m$,

$$
\lim _{n \rightarrow \infty}\left\|\left|f_{m}\right| \wedge\left|f_{n}\right|\right\|=0
$$

Now, by a standard disjointification argument, we may pass to a further subsequence so that, for some disjoint sequence $\left\{h_{n}\right\}_{n=1}^{\infty}$,

$$
\lim _{n \rightarrow \infty}\left\|f_{n}-h_{n}\right\|=0 .
$$

The conclusion follows on passing to yet another subsequence.

(2) Suppose

$$
\inf _{n} \int\left|f_{n}^{*}\right| d \mu=0
$$

Arguing as in (1) we may pass to a subsequence and assume that $\left\{f_{n}^{*}\right\}_{n=1}^{\infty}$ is strongly equivalent to a disjoint sequence $\left\{h_{n}^{*}\right\}_{n=1}^{\infty}$. Further $\left[h_{n}^{*}\right]_{n=1}^{\infty}$ is complemented in $Z^{*}$. If $A_{n}=\operatorname{supp} h_{n}^{*}$ and $Q: Z^{*} \rightarrow\left[h_{n}^{*}\right]$ is a projection then set

$$
Q_{1}\left(g^{*}\right)=\sum_{n=1}^{\infty} \chi_{A_{n}} Q\left(g^{*} \chi_{A_{n}}\right)
$$

Then $\left\|Q_{1}\right\| \leq\|Q\|$ and $Q_{1}$ is also a projection (cf. $[15,1 . c .8]$ for the discrete version). Now

$$
Q_{1}\left(g^{*}\right)=\sum_{n=1}^{\infty}\left(\int_{\Omega} g^{*} h_{n} d \mu\right) h_{n}^{*}
$$

for a disjoint sequence $\left\{h_{n}\right\}_{n=1}^{\infty}$ in $Z$. Then $\left\{h_{n}\right\}_{n=1}^{\infty}$ is equivalent to $\left\{f_{n}\right\}_{n=1}^{\infty}$ and, as $\left[h_{n}\right]_{n=1}^{\infty}$ is complemented, it is strongly equivalent.

(3) Suppose $\delta>0$ and

$$
\int_{\Omega}\left|f_{n}\right| d \mu \geq \delta, \quad \int_{\Omega}\left|f_{n}^{*}\right| d \mu \geq \delta, \quad n=1,2, \ldots
$$

Then, by Khintchine's inequality, for $a_{1}, \ldots, a_{n} \in \mathbb{R}, n=1,2, \ldots$,

$$
\sup _{\varepsilon_{i}= \pm 1} \int_{\Omega}\left|\sum_{i=1}^{n} a_{i} \varepsilon_{i} f_{i}\right| d \mu \geq \frac{\delta}{\sqrt{2}}\left(\sum_{i=1}^{n}\left|a_{i}\right|^{2}\right)^{1 / 2}
$$

and so, for some $\gamma>0$ independent of $n$,

$$
\left\|\sum_{i=1}^{n} a_{i} f_{i}\right\| \geq \gamma\left(\sum_{i=1}^{n}\left|a_{i}\right|^{2}\right)^{1 / 2}
$$

and similarly we can assume

$$
\left\|\sum_{i=1}^{n} a_{i} f_{i}^{*}\right\| \geq \gamma\left(\sum_{i=1}^{n}\left|a_{i}\right|^{2}\right)^{1 / 2} .
$$


Now there exists $g^{*} \in Z^{*}$ with $\left\|g^{*}\right\|=1$ and

$$
\sum_{i=1}^{n} a_{i} \int_{\Omega} g^{*} f_{i} d \mu=\left\|\sum_{i=1}^{n} a_{i} f_{i}\right\| .
$$

If $P$ is the projection of $Z$ onto $\left[f_{n}\right]_{n=1}^{\infty}$ then

$$
\left\|P^{*}\right\| \geq\left\|P^{*}\left(g^{*}\right)\right\| \geq \gamma\left(\sum_{i=1}^{n}\left|\int_{\Omega} g^{*} f_{i} d \mu\right|^{2}\right)^{1 / 2}
$$

and hence

$$
\left\|\sum_{i=1}^{n} a_{i} f_{i}\right\| \leq \gamma^{-1}\|P\|\left(\sum_{i=1}^{n}\left|a_{i}\right|^{2}\right)^{1 / 2}
$$

so that $\left\{f_{i}\right\}_{i=1}^{\infty}$ is equivalent to the $l_{2}$-basis.

From now on we assume that $Z$ is a Banach function space over a nonatomic probability measure space $(\Omega, \Sigma, \mu)$ with nontrivial cotype. We suppose $Z=X \oplus Y$ and $P_{X}$ and $P_{Y}$ are the respective projections.

LEMMA 2.6. If $X$ contains no subspace isomorphic to $l_{2}$, or if $Z$ has nontrivial type and $X$ contains no complemented copy of $l_{2}$ then $X$ satisfies:

(+) For every $f \in Z$ and $\varepsilon>0$ there exists $g \in Z$ and $A \in \Sigma$ so that

(a) $|g|=|f|$.

(b) $\left\|g \cdot \chi_{A}\right\|<\varepsilon$.

(c) $\left\|\left(P_{X}(g)\right) \chi_{\Omega \sim A}\right\|<\varepsilon$.

(d) $\int_{\Omega}\left|P_{X}(g)\right| d \mu<\varepsilon$.

PROOF. Let us consider the first case. If $f \in Z$ and $f \neq 0$ then, by Lemma 2.4, we can find $f_{n} \in Z, n=1,2, \ldots$ with $\left|f_{n}\right|=|f|$, for all $n$, and $\left\{f_{n}\right\}_{n=1}^{\infty}$ equivalent to the $l_{2}$-basis. We show

$$
\inf _{n} \int_{\Omega}\left|P_{X}\left(f_{n}\right)\right| d \mu=0 .
$$

Indeed, if not then, for some $\delta>0$,

$$
\int_{\Omega}\left|P_{X}\left(f_{n}\right)\right| d \mu \geq \delta, \quad n=1,2, \ldots
$$

By passing to a subsequence, we may suppose $\left\{P_{X}\left(f_{n}\right)\right\}_{n=1}^{\infty}$ is basic in $X$ (since $\left\|P_{X}\left(f_{n}\right)\right\| \geq \delta$ but $P_{X}\left(f_{n}\right) \stackrel{w}{\rightarrow} 0$ as $\left.n \rightarrow \infty\right)$. Now, by a theorem of D. J. Aldous and D. H. Fremlin [1], we can pass to a further subsequence and assume that, for some $c>0$,

$$
\int_{\Omega}\left|\sum_{i=1}^{n} a_{i} P_{X}\left(f_{i}\right)\right| d \mu \geq c\left(\sum_{i=1}^{n}\left|a_{i}\right|^{2}\right)^{1 / 2},
$$

for $a_{1}, \ldots, a_{n} \in \mathbb{R}$ and all $n$. Thus

$$
\left\|\sum_{i=1}^{n} a_{i} P_{X}\left(f_{i}\right)\right\| \geq c\left(\sum_{i=1}^{n}\left|a_{i}\right|^{2}\right)^{1 / 2}
$$


However,

$$
\begin{aligned}
\left\|\sum_{i=1}^{n} a_{i} P_{X}\left(f_{i}\right)\right\| & \leq\left\|P_{X}\right\|\left\|\sum_{i=1}^{n} a_{i} f_{i}\right\| \\
& \leq K\left\|P_{X}\right\|\left(\sum_{i=1}^{n}\left|a_{i}\right|^{2}\right)^{1 / 2},
\end{aligned}
$$

for some constant $K$. Thus $\left\{P_{X} f_{n}\right\}_{n=1}^{\infty}$ is equivalent to the $l_{2}$-basis which is a contradiction.

If, in addition $Z$ has nontrivial type, then we can repeat the argument at the end of Lemma 2.5 since $\left\{P_{X}\left(f_{n}\right)\right\}_{n=1}^{\infty}$ is equivalent to $l_{2}$-basis in both $Z$ and $L_{1}(\mu)$. We then can pass to a further subsequence and obtain that $\left[P_{X} f_{n}\right]_{n=1}^{\infty}$ is complemented which is also a contradiction.

In either case we may now assume $\lim _{n \rightarrow \infty} \int_{\Omega}\left|P_{X}\left(f_{n}\right)\right| d \mu=0$. Let $A_{n}=\{\omega \in$ $\left.\Omega:\left|P_{X}\left(f_{n}\right)\right| \geq \varepsilon / 3\right\}$. Then $\mu\left(A_{n}\right) \rightarrow 0$ and so $\left\|f \chi_{A_{n}}\right\| \rightarrow 0$, as $n \rightarrow \infty$. Also

$$
\left\|P_{X}\left(f_{n}\right) \cdot \chi_{\Omega \sim A_{n}}\right\| \leq 2\left\|P_{X}\left(f_{n}\right) \cdot \chi_{\Omega \sim A_{n}}\right\|_{\infty}<\varepsilon .
$$

Hence, for large enough $n$, we may take $g=f_{n}$ and $A=A_{n}$ and (+) will hold.

LEMMA 2.7. Suppose $X$ satisfies $(+)$ and $\left\{f_{n}\right\}_{n=1}^{\infty}$ is a normalized disjoint sequence in $Z$. Then $\left\{f_{n}\right\}_{n=1}^{\infty}$ has a subsequence strongly equivalent to a basic sequence in $Y$.

Proof. Use (+) to pick $h_{n} \in Z, A_{n} \in \Sigma$ so that $\left\|h_{n} \chi_{A_{n}}\right\| \leq 1 / n, \| P_{X}\left(h_{n}\right)$. $\chi \Omega \sim A_{n} \| \leq 1 / n$ and $\int_{\Omega}\left|P_{X}\left(h_{n}\right)\right| d \mu \leq 1 / n$, for all $n$. Let us put $g_{n}=h_{n} \chi \Omega \sim A_{n}-$ $P_{X}\left(h_{n}\right) \chi_{A_{n}}$. Then $\left\{g_{n}\right\}_{n=1}^{\infty}$ is a bounded sequence in $Z$. If $m \leq n$

$$
\int_{\Omega}\left|g_{m}\right| \wedge\left|P_{X}\left(h_{n}\right)\right| \chi_{A_{n}} d \mu \leq \frac{1}{n}
$$

so that

$$
\lim _{n \rightarrow \infty}\left\|\left|g_{m}\right| \wedge\left|g_{n}\right|\right\|=0 .
$$

Now there is a subsequence $\left\{g_{n_{k}}\right\}_{k=1}^{\infty}$ of $\left\{g_{n}\right\}_{n=1}^{\infty}$ and a disjoint sequence $\left\{B_{k}\right\}_{k=1}^{\infty}$ of sets in $\Sigma$ so that

$$
\lim _{k \rightarrow \infty}\left\|g_{n_{k}}-g_{n_{k}} \chi_{B_{k}}\right\|=0 .
$$

Let $C_{k}=\left(\operatorname{supp} f_{n_{k}}\right) \cap B_{k} \cap\left(\Omega \sim A_{n_{k}}\right)$, and define $\phi \in L_{\infty}$ by

$$
\psi(\omega)= \begin{cases}h_{n_{k}}(\omega) / f_{n_{k}}(\omega), & \omega \in C_{k}, \\ 0, & \omega \in \Omega \sim \bigcup_{k=1}^{\infty} C_{k} .\end{cases}
$$

Define $A: Z \rightarrow Z$ by $A(f)=\phi f$. First we note that $A\left(f_{n_{k}}\right)=h_{n_{k}} \chi_{C_{k}}$ and $\| h_{n_{k}}$ $h_{n_{k}} \chi_{C_{k}}\|\leq\| g_{n_{k}}-g_{n_{k}} \chi_{B_{k}} \| \rightarrow 0$, as $k \rightarrow \infty$.

Thus

$$
\lim _{k \rightarrow \infty}\left\|A f_{n_{k}}-h_{n_{k}}\right\|=0 \text {. }
$$

Now $P_{X}\left(g_{n}\right)=P_{X}\left(h_{n}-h_{n} \cdot \chi_{A_{n}}\right)-P_{X}\left(P_{X} h_{n}-\left(P_{X} h_{n}\right) \cdot \chi_{\Omega-A_{n}}\right)$ so that

$$
\left\|P_{X}\left(g_{n}\right)\right\| \leq 2\left\|P_{X}\right\| / n \text {. }
$$


Thus $\left\|P_{Y}\left(g_{n}\right)-g_{n}\right\| \rightarrow 0$. Also

$$
\left\|P_{Y}\left(\left(P_{X} h_{n}\right) \cdot \chi_{A_{n}}\right)\right\|=\left\|P_{Y}\left(\left(P_{X} h_{n}\right) \cdot \chi_{\Omega-A_{n}}\right)\right\| \leq\left\|P_{Y}\right\| / n \rightarrow 0
$$

so that

$$
\left\|P_{Y}\left(g_{n}\right)-P_{Y}\left(h_{n} \cdot \chi_{\Omega-A_{n}}\right)\right\| \rightarrow 0 \quad \text { and } \quad\left\|P_{Y}\left(g_{n}\right)-P_{Y}\left(h_{n}\right)\right\| \rightarrow 0, \quad \text { as } n \rightarrow \infty .
$$

We conclude that

$$
\lim _{k \rightarrow \infty}\left\|g_{n_{k}}-P_{Y}\left(h_{n_{k}}\right)\right\|=0 .
$$

Next note $A\left(g_{n_{k}}\right)=f_{n_{k}} \cdot \chi_{\left(\Omega-A_{n_{k}}\right) \cap B_{k}}$ and so

$$
\begin{aligned}
\left\|A\left(g_{n_{k}}\right)-f_{n_{k}}\right\| & \leq 1 / n_{k}+\left\|h_{n_{k}} \chi_{\left(\Omega-A_{n_{k}}\right) \sim B_{k}}\right\| \\
& \leq 1 / n_{k}+\left\|g_{n_{k}}-g_{n_{k}} \chi_{B_{k}}\right\| \rightarrow 0 \quad \text { as } k \rightarrow \infty .
\end{aligned}
$$

Combining these remarks we have

$$
\lim _{k \rightarrow \infty}\left\|P_{Y} A f_{n_{k}}-g_{n_{k}}\right\|=0, \quad \lim _{k \rightarrow \infty}\left\|A g_{n_{k}}-f_{n_{k}}\right\|=0
$$

so that

$$
\lim _{k \rightarrow \infty}\left\|A P_{Y} A f_{n_{k}}-f_{n_{k}}\right\|=0 .
$$

Thus, for some further subsequence $\left\{f_{n}^{\prime}\right\}_{n=1}^{\infty},\left\{f_{n}^{\prime}\right\}_{n=1}^{\infty}$ is strongly equivalent to $\left\{P_{Y} A\left(f_{n}^{\prime}\right)\right\}_{n=1}^{\infty}$ which is a basic sequence in $Y$.

LEMMA 2.8. Suppose $X$ has the property that no basic sequence in $X$ is strongly equivalent to a disjoint sequence in $Z$. Then $Z$ embeds into $Y^{n}=Y \oplus \cdots \oplus Y(n$ times), for some $n \in \mathbb{N}$.

ProOF. First we note that the norm on $X$ must be equivalent to the $L_{1}$-norm i.e., for some $C$, we have

$$
\|f\| \leq C \int_{\Omega}|f| d \mu, \quad f \in X .
$$

Otherwise, we can find a sequence $\left\{f_{n}\right\}_{n=1}^{\infty} \subset X$ with $\left\|f_{n}\right\|=1$ and

$$
\lim _{n \rightarrow \infty} \int_{\Omega}\left|f_{n}\right| d \mu=0
$$

Then $\lim _{n \rightarrow \infty}\left\|\left|f_{m}\right| \wedge\left|f_{n}\right|\right\|=0$ and so $\left\{f_{n}\right\}_{n=1}^{\infty}$ has a subsequence strongly equivalent to a disjoint sequence.

Next we note that $X$ must be reflexive. For otherwise $l_{1}$ must embed complementably into $X$, and $Z$ must contain a disjoint sequence equivalent to the $l_{1}$-basis whose closed linear span is complemented.

Thus the unit ball of $X$ is weakly compact and so, for any $\varepsilon>0$, there exists $\delta>0$ so that if $f \in X,\|f\| \leq 1$ and $A \in \Sigma$ with $\mu(A)<\delta$ then $\int_{A}|f| d \mu<\varepsilon$. Choose $\delta$ corresponding to $\varepsilon=1 / 2 C$ and partition $\Omega$ into $n$ disjoint sets $\Omega_{k}, 1 \leq k \leq n$, with $\mu\left(\Omega_{k}\right)<\delta$. Let $Z_{k}=Z \mid \Omega_{k}$. 
If $f \in Z_{k}$ then

$$
\begin{aligned}
\frac{1}{2 C}\left\|P_{X}(f)\right\| & \geq \int_{\Omega_{k}}\left|P_{X}(f)\right| d \mu \\
& =\int_{\Omega}\left|P_{X}(f)\right| d \mu-\int_{\Omega \sim \Omega_{k}}\left|P_{X}(f)\right| d \mu \\
& \geq \frac{1}{C}\left\|P_{X}(f)\right\|-\int_{\Omega \sim \Omega_{k}}\left|P_{Y}(f)\right| d \mu \\
& \geq \frac{1}{C}\left\|P_{X}(f)\right\|-\left\|P_{Y}(f)\right\| .
\end{aligned}
$$

Hence,

and

$$
\left\|P_{Y}(f)\right\| \geq \frac{1}{2 C}\left\|P_{X}(f)\right\| \geq \frac{1}{2 C}\left(\|f\|-\left\|P_{Y}(f)\right\|\right)
$$

$$
\left\|P_{Y}(f)\right\| \geq \frac{1}{1+2 C}\|f\| .
$$

Hence, $Z_{k}$ embeds in $Y$ and $Z \approx Z_{1} \oplus \cdots \oplus Z_{n}$ embeds into $Y^{n}$.

Now we complete the proof of Theorem 2.2. We may suppose by Lemma 2.3 that $Z$ is a Banach function space.

ProOF OF 2.2(a). By Lemma 2.6, $X$ satisfies $(+)$ and so by Lemma 2.7, $X$ satisfies the hypotheses of Lemma 2.8. Thus $X$ embeds into $Y^{n}$ and hence $\operatorname{dim} X<$ $\infty$.

PROOF OF 2.2(b). Similar.

PROOF OF 2.2(c). If $Y$ contains a complemented copy of $l_{2}$ this reduces to case (b). Otherwise, we can assume $Y$ contains no complemented copy of $l_{2}$. Thus $Y$ satisfies (+), but by Lemma 2.5 there is a basic sequence in $Y$ strongly equivalent to a disjoint sequence in $Z$. Lemma 2.7 then completes the proof.

The statement of Theorem 2.1 requires that $Z$ be a Banach lattice with some nontrivial cotype. The purpose of the following example is to show that this assumption is not redundant.

EXAMPLE 2.9. There exists a nonatomic order continuous Banach lattice $Z$ which decomposes as a direct sum $Z=X \oplus Y$ and $X$ and $Y$ are infinite dimensional nontotally incomparable spaces.

The idea is to construct an order continuous Banach function space $X$ on $[0,1]$ which contains no isomorphic copy of $l_{2}$. Then $Z=X \oplus L_{2}(1,2)$ provides the desired counter-example.

As $X$ we shall take a separable Orlicz function space $H_{M}(0,1)$ which is considerably "smaller" than the space $H_{N}(0,1)$ with $N(x)=\left(e^{x^{2}}-1\right) /(e-1)$. The space $H_{N}(0,1)$ is, by [21], the smallest rearrangement invariant space in which the Rademacher functions span $l_{2}$. More precisely, let e.g. $M(x)=\left(e^{x^{4}}-1\right) /(e-1)$ and consider the space $H_{M}(0,1)$ of all measurable functions $f$ on $[0,1]$ so that

$$
\int_{0}^{1} M\left(\frac{|f(t)|}{\lambda}\right) d t<\infty
$$

for every $\lambda>0$. The norm in $H_{M}(0,1)$ is defined, as usual, by

$$
\|f\|_{M}=\inf \left\{\lambda>0 ; \int_{0}^{1} M\left(\frac{|f(t)|}{\lambda}\right) d t \leq 1\right\} .
$$


It is well known that the simple functions are dense in $H_{M}(0,1)$ and therefore this space is a separable order continuous lattice. Suppose now that $H_{M}(0,1)$ contains a subspace $V$ isomorphic to $l_{2}$. Then, by [6], either there exists a constant $\alpha>0$ so that $\mu\left\{t \in[0,1] ;|f(t)| \geq \alpha\|f\|_{M}\right\} \geq \alpha$, for all $f \in V$, or $V$ contains a normalized sequence which is equivalent to a sequence of mutually disjoint norm one functions in $H_{M}(0,1)$.

In the first case,

$$
\|f\|_{2} \geq \alpha^{3 / 2}\|f\|_{M}, \quad f \in V .
$$

On the other hand, a simple calculation shows that, for $f \geq 1$ and $x \geq 0, x^{p} \leq$ $(p / 4 e)^{p / 4} e^{x^{r}}$. Hence, there exists a constant $C$ independent of $p$ so that

$$
\|f\|_{p} \leq C p^{1 / 4}\|f\|_{M}, \quad f \in H_{M}(0,1)
$$

i.e. on $V$ the $\|\cdot\|_{2}$ and $\|\cdot\|_{M}$-norms are equivalent. Let $R$ be the orthogonal projection from $L_{2}(0,1)$ onto $V$. Then, for any $p>2$ and $g \in L_{p}(0,1)$, we have

$$
\|R(g)\|_{p} \leq C p^{1 / 4}\|R(g)\|_{M} \leq C p^{1 / 4} \alpha^{-3 / 2}\|R(g)\|_{2} \leq C p^{1 / 4} \alpha^{-3 / 2}\|g\|_{p},
$$

i.e. the norm of $R$ as a projection in $L_{p}(0,1)$ is $\leq C^{\prime} p^{1 / 4}$, for all $p>2$ and some constant $C^{\prime}$ independent of $p$. This implies that the factorization constant $\gamma_{p}\left(l_{2}\right)$ is $\leq C^{\prime \prime} p^{1 / 4}$, for some $C^{\prime \prime}$, while in fact, by [8], $\gamma_{p}\left(l_{2}\right)$ behaves like $p^{1 / 2}$, when $p \rightarrow \infty$. This contradiction completes the proof in the first case.

We consider now the case when $V$ contains a normalized sequence equivalent to a sequence of mutually disjoint functions in $H_{M}(0,1)$. This situation, however, is again contradictory since any such sequence of norm one disjoint functions in $H_{M}(0,1)$ contains a subsequence equivalent to the unit vector basis of $c_{0}$. This fact is known and can be deduced easily in the following way. Let $\left\{h_{n}\right\}_{n=1}^{\infty}$ be a normalized sequence of mutually disjoint elements of $H_{M}(0,1)$ and assume, as we clearly may by passing to a subsequence, that there are sets $\left\{B_{n}\right\}_{n=1}^{\infty}$ such that $\left\|h_{n}-h_{n} \cdot \chi_{B_{n}}\right\| \leq 2^{-(n+1)}$ and $\left|h_{n}(t)\right| \geq 2^{n+1}$, for $t \in B_{n}$ and all $n$. Since $e^{a x} \leq 2 e^{x} / A$, for $0<a<1 / 2$ and $x \geq A$, it follows that, whenever $0<\left|a_{n}\right|<1 / 2$, for all $n$,

$$
\int_{0}^{1} M\left(\left|\sum_{n=1}^{\infty} a_{n} h_{n} \cdot \chi_{B_{n}}\right|\right) d t=\sum_{n=1}^{\infty} \int_{B_{n}} \frac{e^{\left|a_{n} h_{n}\right|_{-1}^{4}}}{e-1} d t \leq 1
$$

i.e.

$$
\left\|\sum_{n=1}^{\infty} a_{n} h_{n} \cdot \chi_{B_{n}}\right\|_{M} \leq 1 \text { and }\left\|\sum_{n=1}^{\infty} a_{n} h_{n}\right\|_{M} \leq 2 .
$$

It is highly likely that Theorem 2.2 can be improved in the case when $Z$ has nontrivial type. Note however that the example $L_{1}(0,1) \oplus L_{2}(1,2)$ shows that Theorem 2.2(a) requires some condition on $X$.

We conclude this section with an analogue of Theorem 1.7.

THEOREM 2.10. Let $X$ be a Banach space with nontrivial type and assume that $1<p<\infty$. Suppose $X \oplus l_{p}$ is isomorphic to a Banach lattice $Z$. Then $X$ is isomorphic to a Banach lattice.

REMARK. The case $p=1$ can be obtained from results in the next section and the case of $c_{0}$ then would follow by duality. We leave the reader to fill in the details. 
PROOF. If $X$ contains a complemented copy of $l_{p}$, then $X \approx Z$ and the theorem is immediate. Assume therefore that every bounded operator $T: X \rightarrow l_{p}$ is strictly singular. $Z$ can then be decomposed into two bands, $Z_{1}$ and $Z_{2}$ so that $Z_{1}$ is atomic and $Z_{2}$ is nonatomic. By Theorem $\mathrm{A}, Z_{1} \approx X_{1} \oplus Y_{1}$ and $Z_{2} \approx X_{2} \oplus Y_{2}$, where $X_{1}$ and $X_{2}$ are complemented subspaces of $X$ and $Y_{1}, Y_{2}$ are complemented subspaces of $l_{p}$.

Assume first $\operatorname{dim} Y_{2}=\infty$, so that $Y_{2} \approx l_{p}$. Then Theorem 2.2(c) implies $\operatorname{dim} X_{2}<\infty$ and so $Z_{2} \approx l_{p}$ (which implies $p=2$ ). Then $X \oplus l_{p}$ has a possibly uncountable unconditional basis. However, the copy of $l_{p}$ can be supported only on countably many basis elements and so we can write $X=W_{1} \oplus W_{2}$, where $W_{1}$ has an unconditional basis, $W_{2}$ is separable and $W_{2} \oplus l_{p}$ has an unconditional basis. Thus, by Theorem 1.7, $X$ is isomorphic to a Banach lattice.

Assume then $\operatorname{dim} Y_{2}<\infty$. Thus $l_{p}$ embeds complementably in $Z_{1}$ and so $Y_{1} \approx l_{p}$. Note that if $\operatorname{dim} Z_{2}=0$, then arguing as above the result follows from Theorem 1.7. Assume therefore $\operatorname{dim} Z_{2}>0$ so that $Z_{2} \approx l_{2} \oplus Z_{2}$.

Now $Z \approx\left(Z_{2} \oplus X_{1}\right) \oplus l_{p} \approx X \oplus l_{p}$ and so by Theorem $\mathrm{A}, Z_{2} \oplus X_{1} \approx U_{1} \oplus V_{1}, l_{p} \approx$ $U_{2} \oplus V_{2}$, where $X_{1} \approx U_{1} \oplus U_{2}$ and $l_{p} \approx V_{1} \oplus V_{2}$. Clearly $\operatorname{dim} V_{1}<\infty$ and $\operatorname{dim} U_{2}<\infty$. However, $\left(Z_{2} \oplus X_{1}\right) \oplus U_{2} \approx X \oplus V_{1}$ and $\left(Z_{2} \oplus X_{1}\right) \oplus U_{2} \approx Z_{2} \oplus X_{1}$ since $Z_{2} \approx Z_{2} \oplus l_{2}$. Thus $Z_{2} \oplus X_{1} \approx X$.

Again from Theorem 1.7, $X_{1}$ is isomorphic to a Banach lattice and so the theorem is proved.

3. Banach lattices containing complemented copies of $L_{1}$-spaces. The object of this section is to study different situations in which an $L_{1}$-space embeds complementably into a Banach lattice. We consider first lattices which contain complemented subspaces isomorphic to $l_{1}$, in the spirit of Theorem 2.10.

THEOREM 3.1. Let $Z$ be a nonatomic order continuous Banach lattice and suppose that $Z=X \oplus Y$, with $Y$ being isomorphic to $l_{1}$. Then $X$ is isomorphic to $Z$.

ProOF. It clearly suffices to show that $X$ contains a complemented subspace which is isomorphic to $l_{1}$.

Since $Z$ is order continuous and contains an isomorphic copy of $l_{1}$ there exists a sequence $\left\{u_{k}\right\}_{k=1}^{\infty}$ of mutually disjoint elements in $Z$ which is equivalent to the unit vector basis of $l_{1}$ (this fact is well known; cf. [26]). Then, for each $k$, we use Lemma 2.3 in order to construct a sequence $\left\{u_{k, n}\right\}_{n=1}^{\infty}$ which converges weakly to zero and so that $\left|u_{k, n}\right|=\left|u_{k}\right|$, for all $n$. If $P_{X}$ and $P_{Y}$ denote the corresponding projections from $Z$ onto $X$, respectively $Y$, then $P_{Y}\left(u_{k, n}\right) \stackrel{w}{\rightarrow} 0$, as $n \rightarrow \infty$. However, since $l_{1}$ has the Schur property it follows that $\left\|P_{Y}\left(u_{k, n}\right)\right\| \rightarrow 0$, as $n \rightarrow \infty$. Choose now an integer $n(k)$ such that $\left\|P_{Y}\left(u_{k, n(k)}\right)\right\|<2^{-(k+3)}$, for all $k$, and notice that $\left\{u_{k, n(i)}\right\}_{k=1}^{\infty}$ is equivalent to the unit vector basis of $l_{1}$ and its span is complemented in $Z$. The above choice of $n(k)$ shows that so is $\left\{P_{X}\left(u_{k, n(k)}\right)\right\}_{k=1}^{\infty}$ and this completes the proof.

It was shown in [11, Theorem 3.1] that if a Banach lattice $Z$ contains no isomorphic copy of $c_{0}$ and has a subspace isomorphic to $L_{1}(0,1)$ then $Z$ also has a sublattice which is order isomorphic to $L_{1}(0,1)$. In the next theorems, we consider this situation from different points of view. 
THEOREM 3.2. Let $Z$ be a Banach function space over a probability space $(\Omega, \Sigma, \mu)$ which contains no subspace isomorphic to $c_{0}$. Suppose that $Z=X \oplus Y$, where $X$ does not contain isomorphic copies of $L_{1}(0,1)$ while $Y$ is an $\mathcal{L}_{1}$-space. Then $Z$ has a band $Z_{0}$ which is order isomorphic to an $L_{1}$-space such that its orthogonal complement $Z_{0}^{\perp}$ contains no isomorphic copies of $L_{1}(0,1)$.

Proof. Step I. Our first aim is to construct the band $Z_{0}$. To this end, we call a set $A \in \Sigma$ with $\mu(A)>0$ acceptable provided there exists a lattice homomorphism $S: L_{1}(0,1) \rightarrow Z$ having the form

$$
S(\psi)(\omega)=a(\omega) \psi(\sigma(\omega)), \quad \psi \in L_{1}(0,1),
$$

where $a: \Omega \rightarrow \mathbb{R}$ and $\sigma: \Omega \rightarrow[0,1]$ are measurable functions and $a(\omega)>0$ for $\omega \in A$. Notice that a subset of positive measure of an acceptable set is acceptable and also the countable union $A=\bigcup_{j=1}^{\infty} A_{j}$ of a sequence $\left\{A_{j}\right\}_{j=1}^{\infty}$ of acceptable sets is acceptable. Indeed, if $S_{j}(\psi)(\omega)=a_{j}(\omega) \psi\left(\sigma_{j}(\omega)\right)$ is the lattice homomorphism corresponding to $A_{j}$ then we set

$$
\begin{aligned}
& a(\omega)=a_{j}(\omega) / 2^{j}\left\|S_{j}\right\|, \\
& \sigma(\omega)=\sigma_{j}(\omega),
\end{aligned} \quad \begin{array}{ll}
\omega \in A_{j}, j=1,2, \ldots,
\end{array}
$$

and $a(\omega)=\sigma(\omega)=0$, for $\omega \notin A$. It follows that $S(\psi)(\omega)=a(\omega) \psi(\sigma(\omega)) ; \omega \in$ $\Omega, \psi \in L_{1}(0,1)$ is a lattice homomorphism from $L_{1}(0,1)$ into $Z$ and $a(\omega)>0$ for $\omega \in A$. The above observations show that there exists a maximal acceptable set $\Omega_{0}$ i.e. a subset $\Omega_{0}$ of $\Omega$ having the following properties:

(i) $\Omega_{0}$ is acceptable.

(ii) $\Omega \sim \Omega_{0}$ contains no acceptable subset.

Let $Z_{0}$ be the band of $Z$ generated by $\Omega_{0}$ i.e. $Z_{0}=\left\{f \cdot \chi_{\Omega_{0}} ; f \in Z\right\}$ and $Z_{0}^{\perp}$ its orthogonal complement.

Suppose now that there exists an isomorphism $T$ from $L_{1}(0,1)$ onto a subspace of $Z_{0}^{\perp}$. Then, by Theorem $\mathrm{D}(\mathrm{i})$, there exists also an order isomorphism $S$ from $L_{1}(0,1)$ into $Z_{0}^{\perp}$. Let $J$ denote the formal identity mapping from $Z$ into $L_{1}(\Omega, \Sigma, \mu)$, given by Theorem $\mathrm{C}$. Then $J S$ is a lattice homomorphism from $L_{1}(0,1)$ into $L_{1}\left(\Omega \sim \Omega_{0}, \Sigma_{\mid \Omega \sim \Omega_{0}}, \mu_{\mid \Omega \sim \Omega_{0}}\right)$ and thus by Theorem $\mathrm{D}(\mathrm{ii}), J S(\psi)(\omega)=a(\omega) \psi(\sigma(\omega))$; $\omega \in \Omega \sim \Omega_{0}, \psi \in L_{1}(0,1)$, for suitable $a$ and $\sigma$. Since $S \neq 0$ it follows that $a(\omega)>0$ on a subset $A_{0}$ of positive measure of $\Omega \sim \Omega_{0}$. This contradiction to the maximality of $\Omega_{0}$ shows that $Z_{0}^{\perp}$ contains no subspaces isomorphic to $L_{1}(0,1)$.

Step II. It remains to prove that $Z_{0}$ is order isomorphic to an $L_{1}$-space. To this end, we shall prove first that, for every $\varepsilon>0$ and $z \in Z_{0}$, there exists a function $\psi$ so that $|\psi|=1$ and $\left\|P_{X}(\psi z)\right\|<\varepsilon$.

Since $\Omega_{0}$ is an acceptable set there exists a lattice homomorphism $S_{0}: L_{1}(0,1) \rightarrow$ $Z$ having the form

$$
S_{0}(\psi)(\omega)=a_{0}(\omega) \psi\left(\sigma_{0}(\omega)\right), \quad \omega \in \Omega, \psi \in L_{1}(0,1),
$$

with $a_{0}$ and $\sigma_{0}$ being measurable and $a_{0}(\omega)>0, \omega \in \Omega_{0}$. Fix now $\varepsilon>0$ and $z \in Z_{0}$, and choose a bounded measurable function $g$ on $\Omega_{0}$ such that $\|z-a g\|<$ $\varepsilon / 2\left\|P_{X}\right\|$, where $P_{X}$ and $P_{Y}$ have the usual meaning. Then define the operator $T: L_{1}(0,1) \rightarrow Z$, by setting

$$
T(\psi)(\omega)=g(\omega) S_{0}(\psi)(\omega), \quad \omega \in \Omega, \psi \in L_{1}(0,1)
$$


Since $\|T\| \leq\|g\|_{\infty}\left\|S_{0}\right\|$ it follows that $P_{X} T$ is a bounded operator from $L_{1}(0,1)$ into $X$. By our assumption, $X$ contains no isomorphic copy of $L_{1}(0,1)$ and therefore, by Theorem $\mathrm{D}$ (iii), $P_{X} T$ is not sign preserving. Hence, there exists a mean zero function $\rho \in L_{1}(0,1)$ with $|\rho|=1$ so that $\left\|P_{X} T(\rho)\right\|<\varepsilon / 2$. Then, with the notation $\psi(\omega)=\rho\left(\sigma_{0}(\omega)\right), \omega \in \Omega$, we get

$$
\left\|P_{X}(\psi z)\right\|<\varepsilon / 2+\left\|P_{X} T(\rho)\right\|<\varepsilon
$$

which completes the proof of Step II.

Step III. We shall prove now that $Z_{0}$ is order isomorphic to an $L_{1}$-space. Let $\left\{z_{j}\right\}_{j=1}^{m}$ be a sequence of mutually disjoint elements in $Z_{0}$. Then, by the assertion proved in Step II with $\varepsilon=\left\|\sum_{j=1}^{m} z_{j}\right\| / m$, we find functions $\left\{\psi_{j}\right\}_{j=1}^{m}$ for which $\left|\psi_{j}\right|=1$ and $\left\|P_{X}\left(\psi_{j} z_{j}\right)\right\|<\left\|\sum_{j=1}^{m} z_{j}\right\| / m$, for all $1 \leq j \leq m$. It follows that

$$
\sum_{j=1}^{m}\left\|z_{j}\right\|=\sum_{j=1}^{m}\left\|\psi_{j} z_{j}\right\|<\sum_{j=1}^{m}\left\|P_{Y}\left(\psi_{j} z_{j}\right)\right\|+\left\|\sum_{j=1}^{m} z_{j}\right\| .
$$

For each $1 \leq j \leq m$, let $z_{j}^{*} \in Z^{*}$ be so that $z_{j}^{*}\left(P_{Y}\left(\psi_{j} z_{j}\right)\right)=\left\|P_{Y}\left(\psi_{j} z_{j}\right)\right\|$ and $\left\|z_{j}^{*}\right\|=1$, and consider the operator $W: Y \rightarrow l_{2}^{m}$, defined by

$$
W(f)=\left\{z_{j}^{*}\left(P_{Y}\left(f \cdot \chi_{B_{j}}\right)\right)\right\}_{j=1}^{m}, \quad f \in Y,
$$

where $B_{j}$ denotes the support of $z_{j}$. In order to verify that $W$ is a bounded operator, suppose that $Y$ is an $\mathcal{L}_{1, \lambda}$-space, for some $\lambda>1$. Then, since $l_{1}$ is of cotype 2 with constant $\sqrt{2}$, we get

$$
\begin{aligned}
\|W(f)\|^{2} & =\sum_{j=1}^{m}\left|z_{j}^{*}\left(P_{Y}\left(f \cdot \chi_{B_{j}}\right)\right)\right|^{2} \leq \sum_{j=1}^{m}\left\|P_{Y}\left(f \cdot \chi_{B_{j}}\right)\right\|^{2} \\
& \leq 2 \lambda^{2}\left(\int_{0}^{1}\left\|\sum_{j=1}^{m} r_{j}(t) P_{Y}\left(f \cdot \chi_{B_{j}}\right)\right\|^{2} d t\right),
\end{aligned}
$$

for all $f \in Y$. Hence, $\|W\| \leq \sqrt{2} \lambda\left\|P_{Y}\right\|$.

However, by a famous result due to A. Grothendieck (see e.g. [15, 2.b.6]), every operator from an $\mathcal{L}_{1}$-space to a Hilbert space is absolutely summing. It follows that the absolutely summing norm $\pi_{1}(W)$ of $W$ satisfies

$$
\pi_{1}(W) \leq \sqrt{2} K_{G} \lambda^{2}\left\|P_{Y}\right\|,
$$

where $K_{G}$ stands for Grothendieck's constant. Hence,

$$
\begin{aligned}
\sum_{j=1}^{m}\left\|P_{Y}\left(\psi_{j} z_{j}\right)\right\| & =\sum_{j=1}^{m} z_{j}^{*}\left(P_{Y}\left(\psi_{j} z_{j}\right)\right)=\sum_{j=1}^{m}\left\|W\left(\psi_{j} z_{j}\right)\right\| \\
& \leq \pi_{1}(W) \max _{\varepsilon_{j}= \pm 1}\left\|\sum_{j=1}^{m} \varepsilon_{j} \psi_{j} z_{j}\right\| \leq \sqrt{2} K_{G} \lambda^{2}\left\|P_{Y}\right\| \cdot\left\|\sum_{j=1}^{m} z_{j}\right\| .
\end{aligned}
$$

Therefore,

$$
\sum_{j=1}^{m}\left\|z_{j}\right\| \leq\left(1+\sqrt{2} K_{G} \lambda^{2}\left\|P_{Y}\right\|\right)\left\|\sum_{j=1}^{m} z_{j}\right\|,
$$

which clearly implies that $Z_{0}$ is order isomorphic to an $L_{1}$-space. 
One cannot expect to prove in Theorem 3.2 that $Z_{0}^{\perp}$ is isomorphic to $X$. For instance, if $X=l_{1} \oplus X_{1}$ with $X_{1}$ being a reflexive band of $Z$ and $Y=L_{1}(0,1)$ then we shall get that $Z_{0}=L_{1}(0,1)$ and $Z_{0}^{\perp}=X_{1}$. As we will see later, it is precisely the assumption that $X$ contains no isomorphic copy of $l_{1}$ that is needed in order to conclude that, essentially speaking, $X$ is isomorphic to $Z_{0}^{\perp}$. Before proving this fact, we need a preliminary result.

THEOREM 3.3. Let $Z$ be a Banach function space over a probability space $(\Omega, \Sigma, \mu)$. If $Z$ contains no isomorphic copy of $c_{0}$ and $Z^{* *} / Z$ is an $\mathcal{L}_{1}$-space then either $Z$ has a band $Z_{0}$ which is order isomorphic to an $L_{1}$-space or $Z$ is a dual space.

ProOF. Since $Z$ contains no subspaces isomorphic to $c_{0}$ it is a band of $Z^{* *}$ and, moreover,

$$
P\left(z^{* *}\right)=\bigvee\left\{z \in Z ; 0 \leq z \leq z^{* *}\right\}, \quad 0 \leq z^{* *} \in Z^{* *},
$$

extends to a norm one positive projection from $Z^{* *}$ onto the canonical embedding of $Z$ into $Z^{* *}$. Thus, $Z^{* *}=Z \oplus Z^{\perp}$, where $Z^{\perp}$ denotes the orthogonal complement of $Z$ in $Z^{* *}$. By our assumption, $Z^{\perp}$ is an $\mathcal{L}_{1}$-space. However, it is well known that a lattice, which is an $\mathcal{L}_{1}$-space, is already order isomorphic to an $L_{1}$-space. Therefore, there exists an $L_{1}$-norm $\|\cdot\|_{L}$ on $Z^{\perp}$ which satisfies $C^{-1}\left\|z^{* *}\right\| \leq\left\|z^{* *}\right\|_{L} \leq C\left\|z^{* *}\right\|$, for some $0<C<\infty$ and any $z^{* *} \in Z^{\perp}$. Define now a function $F$ on $Z^{*}$, by setting

$$
F\left(z^{*}\right)=\sup \left\{\left|z^{* *}\left(z^{*}\right)\right|: z^{* *} \in Z^{\perp},\left\|z^{* *}\right\|_{L} \leq 1\right\},
$$

for all $z^{*} \in Z^{*}$. We clearly have

$$
\left|F\left(z^{*}\right)\right| \leq C\left\|z^{*}\right\|, \quad z^{*} \in Z^{*}
$$

and, moreover, the duality between $L_{1}$ and $M$-norms show that $F$ is a seminorm on $Z^{*}$ so that

$$
F\left(z_{1}^{*}+z_{2}^{*}\right)=\max \left(F\left(z_{1}^{*}\right), F\left(z_{2}^{*}\right)\right)
$$

whenever $z_{1}^{*}$ and $z_{2}^{*}$ in $Z^{*}$ satisfy $z_{1}^{*} \wedge z_{2}^{*}=0$.

By using $F$, we define, for each partition $\pi=\left\{B_{k}\right\}_{k=1}^{m}$ of $\Omega$ into sets of positive measure, a function $\beta_{\pi} \in L_{\infty}(\Omega, \Sigma, \mu)$ by

$$
\beta_{\pi}(\omega)=F\left(\chi_{B_{k}}\right) \quad \text { if } \omega \in B_{k}, 1 \leq k \leq m
$$

It is quite clear that $\beta_{\pi} \geq \beta_{\pi^{\prime}}$, whenever the partition $\pi^{\prime}$ refines $\pi$. Therefore, $\left\{\beta_{\pi}\right\}_{\pi}$ is a decreasing net of functions in the lattice $L_{\infty}(\Omega, \Sigma, \mu)$, which is order complete. Consequently, there exists a $\beta \in L_{\infty}(\Omega, \Sigma, \mu)$ so that $\beta=\bigwedge_{\pi} \beta_{\pi}$ and then $\beta_{\pi} \geq \beta$ a.e. for every $\pi$.

We distinguish between two mutually exclusive cases.

Case I. Suppose that $\beta(\omega)>0$ on a subset of $\Omega$ of positive measure and find a $\delta>0$ and a subset $\Omega_{0}$ of $\Omega$ with $\mu\left(\Omega_{0}\right)>0$ so that $\beta(\omega) \geq \delta, \omega \in \Omega_{0}$. We shall prove that in this case the band $Z_{0}=\left\{f \cdot \chi_{\Omega_{0}} ; f \in Z\right\}$ is order isomorphic to an $L_{1}$-space. Let $g \in Z^{*}$ be a simple function of the form $\sum_{i=1}^{m} a_{i} \chi_{A_{i}}$, where $\left\{A_{i}\right\}_{i=1}^{m}$ are mutually disjoint subsets of $\Omega_{0}$ each having positive measure. Then, since $F$ is 
an $M$-seminorm, we obtain

$$
\begin{aligned}
F(g) & =\max _{1 \leq i \leq m}\left|a_{i}\right| \cdot F\left(\chi_{A_{i}}\right) \geq\left(\max _{1 \leq i \leq m}\left|a_{i}\right|\right) \cdot\left(\min _{1 \leq i \leq m} F\left(\chi_{A_{i}}\right)\right) \\
& =\|g\|_{\infty} \cdot \min _{\omega \in \cup_{i=1}^{m} A_{i}} \beta_{\pi}(\omega),
\end{aligned}
$$

for any partition $\pi$ which includes the sets $\left\{A_{i}\right\}_{i=1}^{m}$. Since $\beta_{\pi}(\omega) \geq \beta(\omega)$ a.e. it follows that

$$
C\|g\| \geq F(g) \geq\|g\|_{\infty} \cdot \min _{\omega \in \cup_{i=1}^{m} A_{i}} \beta(\omega) \geq \delta\|g\|_{\infty},
$$

which clearly implies that $C\left\|z^{*}\right\| \geq \delta\left\|z^{*}\right\|_{\infty}$, whenever $z^{*} \in Z^{*}$ is supported by $\Omega_{0}$. Hence, for any $z \in Z_{0}$, we have $\|z\|_{1} \leq\|z\| \leq C \delta^{-1}\|z\|_{1}$. This completes the proof in Case I.

Case II. $\beta(\omega)=0$, for a.e. $\omega \in \Omega$. We shall prove that in this case $Z$ is a conjugate space. Choose first an increasing sequence $\left\{\pi_{n}\right\}_{n=1}^{\infty}$ of partitions of $\Omega$ such that $\beta_{\pi_{n}}(\omega) \rightarrow 0$, as $n \rightarrow \infty$, for a.e. $\omega \in \Omega$. By Egoroff's theorem, there exists a countable partition $\left\{\Omega_{j}\right\}_{j=1}^{\infty}$ of $\Omega$ so that $\mu\left(\Omega \sim \bigcup_{j=1}^{\infty} \Omega_{j}\right)=0$ and $\beta_{\pi_{n}}(\omega) \rightarrow 0$, as $n \rightarrow \infty$, uniformly for $\omega \in \Omega_{j}, j=1,2, \ldots$. For each $j$, let $V_{j}$ be the closure of $L_{\infty}\left(\Omega_{j}, \Sigma_{\mid \Omega_{j}}, \mu_{\mid \Omega_{j}}\right)$ in $Z^{*}$ and $Z_{j}=\left\{f \chi_{\Omega_{j}}: f \in Z\right\}$. Since $Z=\sum_{j=1}^{\infty} \bigoplus Z_{j}$ is a boundedly complete unconditional decomposition (for $Z$ contains no copy of $c_{0}$ ) it would follow that $Z$ is a dual space provided we show that $Z_{j}$ is a conjugate space, for every $j$. This will be achieved by proving that $Z_{j}$ is order isometric to $V_{j}^{*}$. To this end, fix $j$, let $\theta \in V_{j}^{*}$ and find a Hahn-Banach extension $z^{* *}$ of $\theta$ to an element of $Z^{* *}$. If $Z_{j}^{\perp}$ denotes the orthogonal complement of $Z_{j}$ in $Z$ then clearly $Z^{* *}=Z_{j} \oplus Z_{j}^{\perp} \oplus Z^{\perp}$. Hence, $z^{* *}=z+z^{\prime}+z_{0}^{* *}$ with $z \in Z_{j}, z^{\prime} \in Z_{j}^{\perp}$ and $z_{0}^{* *} \in Z^{\perp}$. Then, for $v^{*} \in V_{j}$,

$$
\theta\left(v^{*}\right)=z^{* *}\left(v^{*}\right)=v^{*}\left(z_{j}\right)+z_{0}^{* *}\left(v^{*}\right)
$$

and the proof will be completed once we show that $z_{0}^{* *}\left(v^{*}\right)=0$, whenever $z_{0}^{* *} \in Z^{\perp}$ and $v^{*} \in V_{j}$. However, in view of the definitions of $F$ and $V_{j}$, it suffices to prove that $F$ vanishes on $L_{\infty}\left(\Omega_{j}, \Sigma_{\mid \Omega_{j}}, \mu_{\mid \Omega_{j}}\right)$. In order to verify this fact, notice that, for any measurable subset $A$ of $\Omega_{j}$ and each $n$, we have

$$
F\left(\chi_{A}\right)=\max _{B \in \pi_{n}} F\left(\chi_{A \cap B}\right) \leq \max _{\substack{B \in \pi_{n} \\ A \cap B \neq \varnothing}} F\left(\chi_{B}\right) \leq \max _{\omega \in \Omega_{j}} \beta_{\pi_{n}}(\omega) \rightarrow 0
$$

as $n \rightarrow \infty$.

THEOREM 3.4. Let $Z$ be a Banach function space over a nonatomic probability space $(\Omega, \Sigma, \mu)$. Suppose that $Z=X \oplus Y$, where $X$ is a reflexive space and $Y$ is isomorphic to an $L_{1}$-space. Then there exists a band $Z_{0}$ of $Z$ such that $Z_{0}$ is linearly isomorphic to $Y$ and order isomorphic to an $L_{1}$-space while its orthogonal complement $Z_{0}^{\perp}$ is reflexive and isomorphic to $X$, up to a finite dimensional space.

PROOF. By Theorem 3.2, $Z$ has a band $Z_{0}$ which is order isomorphic to an $L_{1}$-space and such that $Z_{0}^{\perp}$ contains no isomorphic copies of $L_{1}(0,1)$.

We observe now that every operator $T$ from an $L_{1}$-space into a reflexive space is strictly singular since $T$ is weakly compact and $L_{1}$ has the Dunford-Pettis property (thus, the unit ball of any reflexive subspace of $L_{1}$ is mapped by $T$ into a norm-compact set). We may therefore apply Theorem $\mathrm{A}$ and find decompositions 
$X=X_{1} \oplus X_{2}$ and $Y=Y_{1} \oplus Y_{2}$ so that $Z_{0}$ is isomorphic to $X_{1} \oplus Y_{1}$ and $Z_{0}^{\perp}$ to $X_{2} \oplus Y_{2}$. This already implies that $\operatorname{dim} X_{1}<\infty$.

Since $\left(Z_{0}^{\perp}\right)^{* *}$ is isomorphic to $X_{2} \oplus Y_{2}^{* *}$ it follows that $\left(Z_{0}^{\perp}\right)^{* *} / Z_{0}^{\perp}$ is isomorphic to $Y_{2}^{* *} / Y_{2}$. On the other hand, $Y_{2}$ is clearly an $\mathcal{L}_{1}$-space and thus so is $Y_{2}^{* *} / Y_{2}$. By using Theorem 3.3, we conclude that $Z_{0}^{\perp}$ is a dual space (here, we use the fact that $(\Omega, \Sigma, \mu)$ is nonatomic which implies that any nontrivial band of $Z_{0}^{\perp}$ that is order isomorphic to an $L_{1}$-space must contain an isomorphic copy of $L_{1}(0,1)$, a contradiction). Let $X_{2}^{\prime}$ and $Y_{2}^{\prime}$ be subspaces of $Z_{0}^{\perp}$ so that $Z_{0}^{\perp}=X_{2}^{\prime} \oplus Y_{2}^{\prime}, X_{2}^{\prime}$ is isomorphic to $X_{2}$ and $Y_{2}^{\prime}$ to $Y_{2}$. Since $X_{2}^{\prime}$ is reflexive it would be a $w^{*}$-closed subspace of the dual space $Z_{0}^{\perp}$. Hence, $Y_{2}^{\prime}$, which is isomorphic to $Z_{0}^{\perp} / X_{2}^{\prime}$, will be a dual space, too.

Notice now that, since the dual $Z^{*}$ of $Z$ is contained in $L_{1}(\Omega, \Sigma, \mu)$, the set $\left\{\chi_{A} ; A \in \Sigma\right\}$ is weakly compact and its span is dense in $Z$. Hence, $Z$ is a weakly compactly generated (WCG) space and so are its complemented subspaces $Y$ and $Y_{2}$. By using a result of $\mathrm{H}$. P. Rosenthal [23, Corollary 2.2], we obtain that $Y_{2}$ is isomorphic to a complemented subspace of $L_{1}(0,1)$. Thus, by D. R. Lewis and C. Stegall [13], $Y_{2}$ is either isomorphic to $l_{1}$ or $\operatorname{dim} Y_{2}<\infty$. However, in view of Theorem 3.1, the first possibility cannot take place. Hence, $\operatorname{dim} Y_{2}<\infty$. It follows easily that, up to a finite dimensional space, $Z_{0}$ is isomorphic to $Y$ and $Z_{0}^{\perp}$ to $X$. However, $Z_{0}$ contains a complemented subspace isomorphic to $l_{1}$ and therefore $Z_{0}$ is precisely isomorphic to $Y$.

REMARK. Theorem 3.4 remains true even when $(\Omega, \Sigma, \mu)$ is an arbitrary probability space. The proof of this fact uses both Theorems 1.1 and 3.4. Let $Z^{\prime}$ be the band of $Z$ containing all the atoms of $Z$ and $Z^{\prime \prime}$ its orthogonal complement, which is a nonatomic lattice. If $Z=X \oplus Y$ with $Y$ being reflexive and $Y$ isomorphic to an $L_{1}$-space then, by Theorem $\mathrm{A}$, there exist decompositions $X=X^{\prime} \oplus X^{\prime \prime}$ and $Y^{\prime}=Y^{\prime} \oplus Y^{\prime \prime}$ so that $Z^{\prime}$ is isomorphic to $X^{\prime} \oplus Y^{\prime}$ and $Z^{\prime \prime}$ to $X^{\prime \prime} \oplus Y^{\prime \prime}$. The band $Z^{\prime}$ is actually a space with an unconditional basis. Since every operator from $X^{\prime}$ into $Y^{\prime}$ is compact it follows from Theorem 1.1 that $Z^{\prime}=Z_{1}^{\prime} \oplus Z_{2}^{\prime \prime}$, where $Z_{1}^{\prime}$ and $Z_{2}^{\prime \prime}$ are orthogonal bands so that $Z_{1}^{\prime}$ is isomorphic to $X^{\prime}$ and $Z_{2}^{\prime \prime}$ to $Y^{\prime}$. On the other hand, by Theorem 3.4, $Z^{\prime \prime}=Z_{1}^{\prime \prime} \oplus Z_{2}^{\prime \prime}$, where again $Z_{1}^{\prime \prime}$ and $Z_{2}^{\prime \prime}$ are orthogonal bands such that $Z_{1}^{\prime \prime}$ is isomorphic to $X^{\prime \prime}$, up to a finite dimensional space, and $Z_{2}^{\prime \prime}$ is isomorphic to $Y^{\prime \prime}$. Put $Z_{1}=Z_{1}^{\prime} \oplus Z_{1}^{\prime \prime}$ and $Z_{2}=Z_{2}^{\prime \prime} \oplus Z_{2}^{\prime \prime}$. Then $Z=Z_{1} \oplus Z_{2}$, where $Z_{1}$ and $Z_{2}$ are orthogonal bands so that $Z_{1}$ is isomorphic to $X$, up to a finite dimensional space, and $Z_{2}$ to $Y$.

The difficulties encountered in Theorem 3.4 and the remark following it stem from the fact that we do not know whether a reflexive Banach lattice must be isomorphic to its hyperplanes.

Suppose that we replace the assumption made in the above remark that $X$ is reflexive by the assumption that $X$ has nontrivial type. In this case, $Z_{1}$ will be a lattice with nontrivial type and therefore, by Lemma $2.4(\mathrm{c}), Z_{1}$ would contain a complemented copy of $l_{2}$. Thus, $Z_{1}$ will be isomorphic to its hyperplanes which implies the existence of an isomorphism between $Z_{1}$ and $X$. We summarize these conclusions in the following corollary.

COROLLARY 3.5. Let $Z$ be a Banach lattice with a weak unit and suppose that $Z=X \oplus Y$, where $X$ is a subspace with nontrivial type and $Y$ is isomorphic to an 
$L_{1}$-space. Then there exists a band $Z_{0}$ of $Z$ which is isomorphic to $Y$ and so that its orthogonal complement $Z_{0}^{\perp}$ is isomorphic to $X$.

Corollary 3.5 enables us to solve positively a problem raised by $\mathrm{P}$. Wojtaszczyk in [28].

COROLLARY 3.6. If a nonatomic Banach lattice $Z$ is linearly isomorphic to the direct sum $L_{1}(0,1) \oplus L_{2}(0,1)$ then $Z$ is already order isomorphic to $L_{1}(0,1) \oplus$ $L_{2}(0,1)$.

This result means that $L_{1}(0,1) \oplus L_{2}(0,1)$ has, up to isomorphism, a unique structure as a Banach function space on $[0,1]$. Further results on uniqueness of structures in Banach function spaces can be found in [9].

We remark that we can now list all the Banach lattices isomorphic to $L_{1} \oplus L_{2}$. These are $L_{1} \oplus L_{2}, L_{1} \oplus l_{2}, L_{1} \oplus L_{2} \oplus l_{2}, L_{1} \oplus l_{1} \oplus L_{2}, L_{1} \oplus l_{1} \oplus l_{2}, L_{1} \oplus l_{1} \oplus$ $L_{2} \oplus l_{2}, L_{1} \oplus L_{2} \oplus l_{2}^{n}$ for $n \in \mathbb{N}$.

\section{REFERENCES}

1. D. J. Aldous and D. H. Fremlin, Colacunary sequences in L-spaces, Studia Math. 71 (1982), 297-304.

2. J. Bourgain, P. G. Casazza, J. Lindenstrauss and L. Tzafriri, Banach spaces with a unique unconditional basis, up to permutation, Mem. Amer. Math. Soc. No. 322 (1985).

3. I. S. Edelstein and P. Wojtaszczyk, On projections and unconditional bases in direct sums of Banach spaces, Studia Math. 56 (1976), 263-276.

4. P. Enflo and T. Starbird, Subspaces of $L_{1}$ containing $L_{1}$, Studia Math. 65 (1979), 203-225.

5. H. Fakhoury, Representations d'operateurs a valeurs dans $L_{1}(X, \Sigma, \mu)$, Math. Ann. 240 (1979), 203-212.

6. T. Figiel, W. B. Johnson and L. Tzafriri, On Banach lattices and spaces having local unconditional structure with applications to Lorentz function spaces, J. Approx. Theory 13 (1975), 395-412.

7. N. Ghoussoub and H. P. Rosenthal, Martingales, $G_{\delta}$-embeddings and quotients of $L_{1}$, Math. Ann. 264 (1983), 321-332.

8. Y. Gordon, D. R. Lewis and J. R. Retherford, Banach ideals of operators with applications, J. Funct. Anal. 14 (1973), 85-129.

9. W. B. Johnson, B. Maurey, G. Schechtman and L. Tzafriri, Symmetric structures in Banach spaces, Mem. Amer. Math. Soc. No. 217 (1979).

10. N. J. Kalton, The endomorphisms of $L_{p}, 0 \leq p \leq 1$, Indiana Univ. Math. J. 27 (1978), 353-381.

11. __ Embedding $L_{1}$ in a Banach lattice, Israel J. Math. 32 (1979), 209-220.

12. J. L. Krivine, Theoremes de factorisation dans les espaces reticules, Sem. Maurey-Schwartz, Ecole Polyt., Paris, Expose 22-23, 1973-74.

13. D. R. Lewis and C. Stegall, Banach spaces whose duals are isomorphic to $l_{1}(\Gamma)$, J. Funct. Anal. 12 (1973), 177-187.

14. J. Lindenstrauss and A. Pelczynski, Absolutely summing operators in $\mathcal{L}_{p}$-spaces and their applications, Studia Math. 29 (1968), 275-326.

15. J. Lindenstrauss and L. Tzafriri, Classical Banach spaces. I: Sequence spaces, Springer-Verlag, Berlin and New York, 1977.

16. __ Classical Banach spaces. II: Function spaces, Springer-Verlag, Berlin and New York, 1979.

17. H. P. Lotz and H. P. Rosenthal, Embeddings of $C(\Delta)$ and $L^{1}[0,1]$ in Banach lattices, Israel J. Math. 31 (1978), 169-179.

18. B. Maurey, Type et cotype dans les espaces munis des structures locales inconditionnelles, Sem. Maurey-Schwartz, Ecole Polyt., Paris, Expose 24-25, 1973-74. 
19. B. Maurey, Theoremes de factorisation pour les operateurs a valeurs dans un espace $L^{p}$, Asterisque, Soc. Math. France 11 (1974).

20. G. Pisier, Une propriete de stabilite de la classe des espaces ne contenant pas $l^{1}$, C. R. Acad. Sci. Paris 286 (1978), 747-749.

21. V. A. Rodin and E. M. Semyonov, Rademacher series in symmetric spaces, Analyse Math. 1 (1975), 207-222.

22. H. P. Rosenthal, On totally incomparable Banach spaces, J. Funct. Anal. 4 (1969), 167-175.

23. __ On injective Banach spaces and the $L^{\infty}(\mu)$ for finite measure $\mu$, Acta Math. 124 (1970), 205-248.

24. __ Some remarks concerning sign-embeddings, Seminaire d'Analyse Fonct., Univ. Paris VII, 1981-82.

25. __ Embeddings of $L^{1}$ in $L^{1}$, Conference in Modern Analysis and Probability, Contemporary Math., vol. 26, Amer. Math. Soc., Providence, R.I., 1982, pp. 335-349.

26. H. H. Schaefer, Banach lattices and positive operators, Springer-Verlag, Berlin and New York, 1974.

27. P. Wojtaszczyk, On complemented subspaces and unconditional bases in $l_{p} \oplus l_{q}$, Studia Math. 47 (1973), 197-206.

28. __ On projections and unconditional bases in direct sums of Banach spaces. II, Studia Math. 62 (1978), 193-201.

Department of Mathematics, University of Missouri, Columbia, Missouri 65211 (Current address of P. G. Casazza and N. J. Kalton)

Department of Mathematics, The Hebrew University, Jerusalem, IsRael (Current address of L. Tzafriri) 